\title{
Wave Tank Testing of a Pendulum Wave Energy Converter 1:12 Scale Model
}

\author{
Pozzi Nicola*, Bracco Giovanni ${ }^{\dagger}$, Passione Biagio ${ }^{\ddagger}$, \\ Sirigu Sergej Antonello ${ }^{\S}$, Vissio Giacomo " and Mattiazzo Giulianal \\ Department of Mechanical and Aerospace Engineering \\ Politecnico di Torino, Corso Duca Degli Abruzzi 24 \\ Torino 10129, Italy \\ *nicola.pozzi@polito.it \\ †'giovanni.bracco@polito.it \\ ¥biagio.passione@polito.it \\ §sergej.sirigu@polito.it \\ 『giacomo.vissio@polito.it \\ "giuliana.mattiazzo@polito.it \\ Sannino Gianmaria \\ ENEA, Energy and Environment Modeling Unit \\ Via Anguillarese 301 \\ Santa Maria di Galeria - Roma 00123, Italy \\ gianmaria.sannino@enea.it
}

Received 5 September 2016

Accepted 14 January 2017

Published 23 March 2017

\begin{abstract}
Wave Energy is a widespread, reliable renewable energy source. The early study on Wave Energy dates back in the 70's, with a particular effort in the last and present decade to make Wave Energy Converters (WECs) more profitable and predictable. The PeWEC (Pendulum Wave Energy Converter) is a pendulum-based WEC. The research activities described in the present work aim to develop a pendulum converter for the Mediterranean Sea, where waves are shorter, thus with a higher frequency compared to the ocean waves, a characteristic well agreeing with the PeWEC frequency response. The mechanical equations of the device are developed and coupled with the hydrodynamic Cummins equation. The work deals with the design and experimental tank test of a 1:12 scale prototype. The experimental data recorded during the testing campaign are used to validate the numerical model previously described. The numerical model proved to be in good agreement with the experiments.
\end{abstract}

Keywords: Pendulum; wave energy converter; ocean energy; wave power; AQWA; hydrodynamics; scale model; wave tank.

This is an Open Access article published by World Scientific Publishing Company. It is distributed under the terms of the Creative Commons Attribution 4.0 (CC-BY) License. Further distribution of this work is permitted, provided the original work is properly cited. 


\section{Introduction}

Modern research on Wave Energy started in the 70's in Europe and with a particular effort on the last decade [Falcão, 2010, 2013; López et al., 2013; Güney, 2015]. At the time of writing, the interest of Countries is still high with considerable public funding to help the sector to reach its maturity with the development of cost effective, reliable WEC (Wave Energy Converter) [Engström et al., 2009; EU Horizon 2020, 2016; US Department of Energy, 2016]. Furthermore, several typologies of architectures were developed and studied, considering different ways of power extraction, from hydraulic actuators to piezoelectric bimorph [Solovyev and Duong, 2016].

At the Politecnico di Torino, in Italy, the research on Wave Energy started in 2006 with particular focus on the Mediterranean high frequency-low amplitude waves [Bracco et al., 2008]. The high frequency of waves is particularly favorable to the use of inertial devices, where the forces used to generate power are proportional to the incoming excitation frequency [Bracco et al., 2010]. The research activities dealt with the ISWEC system (Inertial Sea Wave Energy Converter), a WEC able to extract power from a rocking hull by means of gyroscopic effects. The use of the gyro allows converting a relatively small rocking motion of the hull into a bigger internal precession motion that is transformed into electric energy.

The main advantages of the gyroscopic technology are the possibility to tune the device on several sea state by varying the gyro speed and control and the intrinsic reliability of the device due to the all-enclosed architecture with no moving parts exposed to the sea environment. The research moved from concept, through 1:45 and 1:8 scaled prototype up to the deployment in August 2015 of a full-scale pilot plant at the Island of Pantelleria, in the Channel of Sicily, in Italy [Bracco et al., 2011, 2015a,b,c; Cagninei et al., 2015].

The ISWEC is an inertial "active" device, since the inertial response can be adapted by varying the gyro speed. Therefore, the device needs to drain a small amount of the produced power to keep the gyroscope in rotation [Sirigu et al., 2016].

A different architecture of inertial WEC that uses the forces produced by a pendulum for use in the Mediterranean Sea was developed. The device is called PeWEC (Pendulum Wave Energy Converter) and does not need to be powered to produce inertial effect, so here it is referred as to "passive" device [Rinaldi et al., 2015]. The "passive" device still maintains the advantages derived from the allenclosed architecture, typical of the inertial harvesters [French, 2006; Trimble et al., 2010; Xie and Cai, 2015].

Clément et al. performed previous work on pendulum energy conversion at the Ecòle Centrale de Nantes with the development of the Searev converter. Cordonnier et al. [2015] described the development of a pendulum-based WEC optimized for the Oceanic site of the Isle of Yeu (France).

The research activities described in the present work aim to develop a pendulum converter for a Mediterranean site, where waves are shorter, thus with a higher frequency and able to activate more efficiently the pendulum (See Sec. 5). The 
design site for the PeWEC is the Island of Pantelleria, one of the most powerful Mediterranean sites [Liberti et al., 2013].

\section{Working Principle}

PeWEC is a pendulum-based floating Wave Energy Converter. This device is mainly composed of a floating hull moored on the seabed and a pendulum connected to the shaft of an electrical generator, which is integral with the hull structure. In other words, the generator shaft constitutes the pendulum hinge. Pendulum, electrical generator and all other apparatus necessary for the device functioning are enclosed in the hull, so they are protected against the corrosive action of sea water and a greater level of durability is guaranteed (see Fig. 1).

The working principle of PeWEC can be explained, from the qualitative point of view, using a bi-dimensional representation, as shown in Fig. 2. In the initial time, the hull and the pendulum are supposed to be at rest. As the waves tilt the hull, it begins its motion along surge, heave and pitch directions. Since pendulum hinge is integral with the hull structure, it moves in the space with it and as consequence, pendulum oscillations are induced. The relative rotation of the pendulum with respect to the hull is used to drive the electrical generator shaft.

The extraction of energy from the system is achieved by damping the pendulum oscillations. The electrical generator (also called Power Take Off, PTO) is controlled to act as a rotary damper coupled to the pendulum.

During the evolution of the system in time, the pendulum exchanges with the hull inertial, Coriolis and gravitational forces and torques. The action of these forces needs to be taken into account during the sizing of the PTO bearings and of the hull structure.

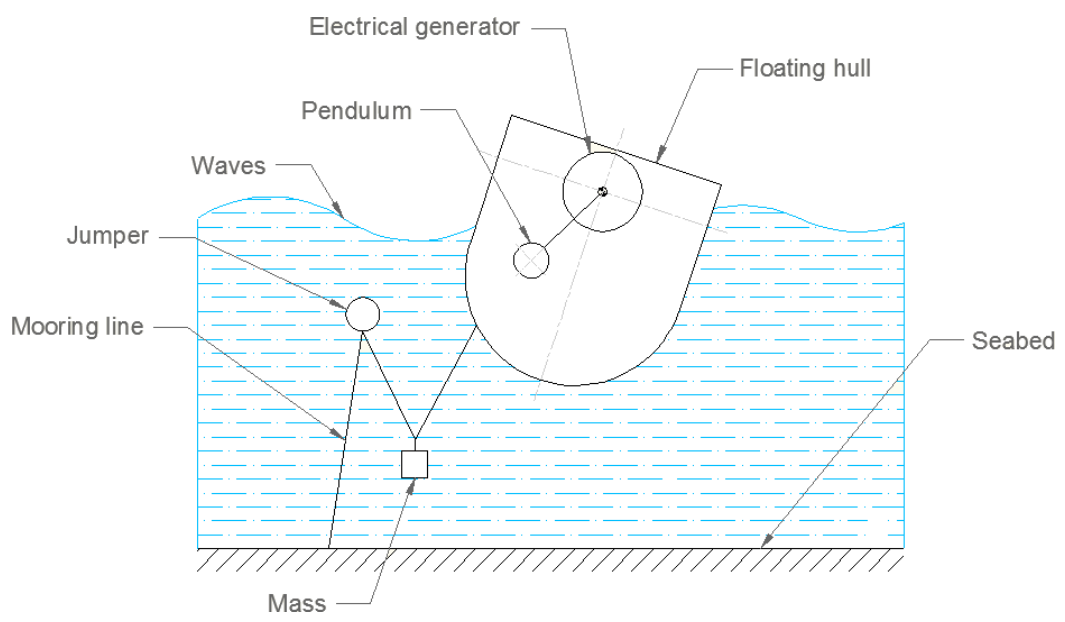

Fig. 1. PeWEC layout. 

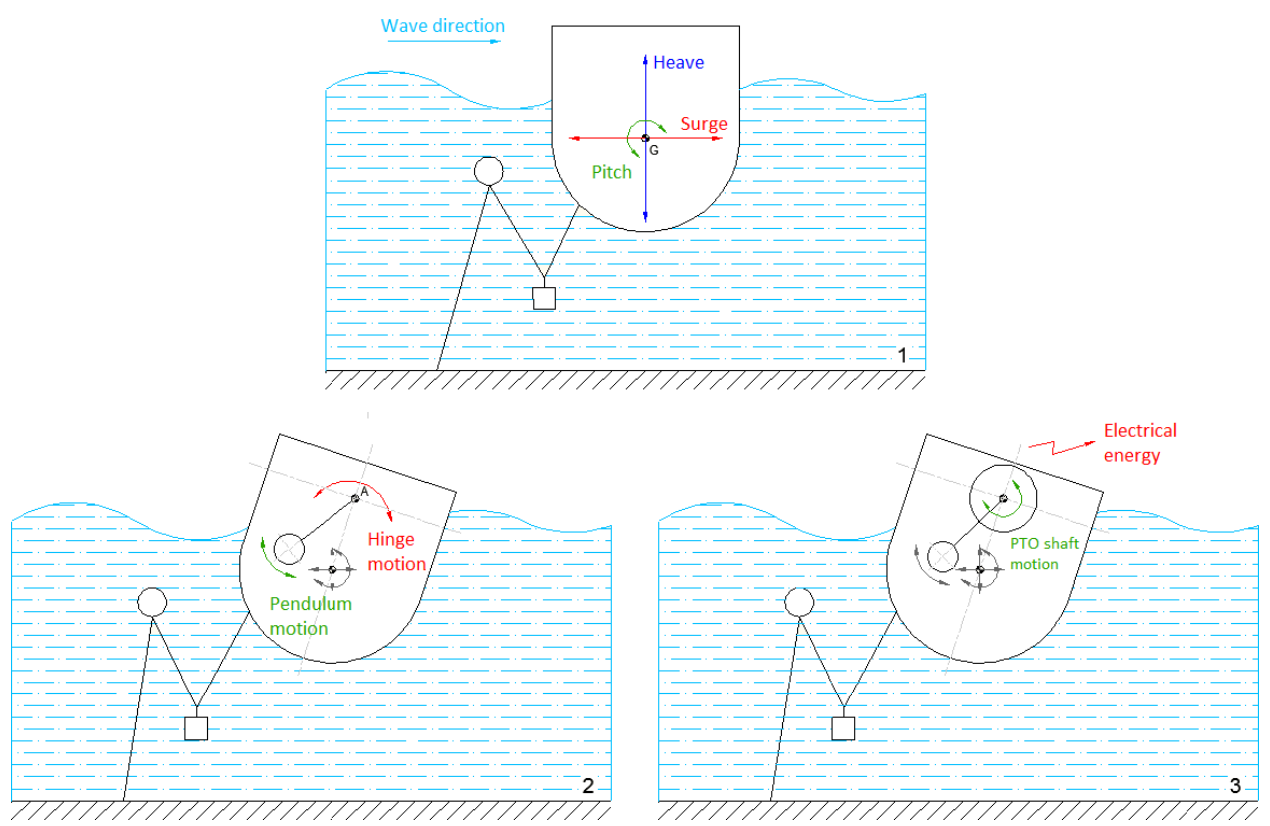

Fig. 2. PeWEC working principle.

As stated before, the floating structure of the device is moored to the seabed in order to keep it in place under the action of the waves. A submerged jumper, a clump weight and three chain sections constitute the mooring line, as shown in Fig. 1. From the physical point of view, this arrangement behaves like a spring. Furthermore, the mooring line is designed such that it does not significantly interfere with the motions of the hull, since they are of primary importance for the activation of the pendulum motion.

The mooring stiffness is particularly influenced by the weight per unit of length of chains, the mass of the clump weight and the length of chain sections. In this case, the chain sections were realized using nylon ropes, which are lighter than steel chains, thus a significant reduction of the mooring stiffness was achieved. Then the clump weight mass, the ropes length and the size of the jumper were optimized through numerical simulations, taking into account the boundary conditions defined by the water depth and the attachment point of the mooring line on the hull. It is important to highlight that the attachment point position was varied during the optimization procedure.

\section{Mechanical Equations}

\subsection{Reference frames}

In Fig. 3, the simplified scheme shown in Fig. 1 was completed with the reference frames and the significant physical points of the system. $G$ is the center of gravity 


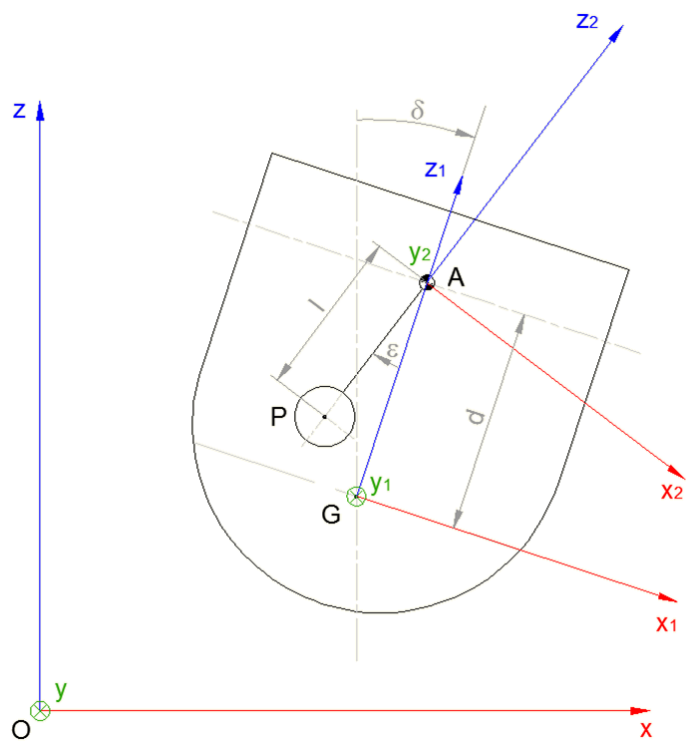

Fig. 3. Simplified scheme of the pendulum system with its reference frames.

of the system, $A$ identifies the position of the pendulum hinge and $P$ is the center of gravity of the pendulum mass. Moreover, let $l$ be the pendulum length and $d$ the distance between $A$ and $G$.

$m_{b}$ is the hull mass and $I_{b}$ its inertia (evaluated with respect to the hull's center of gravity, $G) \cdot m_{p}$ the pendulum mass and $I_{y}$ its inertia evaluated with respect to the mass's center of gravity, $P$.

According to Fig. 3, the following reference frames can be defined:

- $O-x y z$ : right-hand fixed reference frame with origin $O$;

- $G-x_{1} y_{1} z_{1}$ : right-hand reference frame of the floating body with origin coincident with its center of gravity $G$;

- $A-x_{2} y_{2} z_{2}$ : right-hand reference frame of the moving mass with origin coincident with its center of rotation gravity $A$.

The $x$-axis is oriented along the wave direction, with positive direction concordant with respect to the propagation of the wave.

The vertical $z$-axis has positive direction from the bottom upwards, while the direction of the $y$-axis is determined by using right-hand rule. As consequence, it is convenient to choose the same orientation for $x_{1}, y_{1}, z_{1}$ axes and $x_{2}, y_{2}, z_{2}$ axes.

The two-dimensional representation of the system is based on the hypothesis of self-alignment property of the system, with the dominant waves direction. In this way, it is possible to neglect the translation of the system along $y$-axis and the rotation about the $x$-axis. The self-alignment property is mainly due to the mooring line design. The mooring line, as described in Sec. 8, finishes with two 
arms connected to the left and right side of the hull: if the device is not aligned with respect to the wave direction, then a difference in the tensions on the arms is induced, determining a reaction that contributes to the alignment of the device. Under this assumption, the motion of the hull takes place in $O$-xz plane and it can be described by the surge motion $x_{G}$, the heave motion $z_{G}$ and the pitch motion $\delta$ around $y$-axis.

Angular coordinate $\varepsilon$ describes the relative motion between the hull and the inner pendulum.

\subsection{Equation derivation}

The derivation of pendulum dynamic equations was performed coupling the multibody notation commonly used in robotics applications, with the Lagrange approach [Pozzi, 2014].

The Lagrange function is defined as the difference between the kinetic energy $E_{k}$ of the system and its potential energy $E_{p}$.

$$
L=E_{k}-E_{p}
$$

If the Lagrange function of the system is known, then equations of motion are obtained by a direct substitution of its expression into the Euler-Lagrange equation (see Eq. (3.2)). Furthermore, the Euler-Lagrange equation may be completed by introducing the Rayleigh dissipative function $F$, which is a function used to handle the effects of velocity proportional frictional forces. This equation has the following form:

$$
\begin{aligned}
& \frac{d}{d t}\left(\frac{\partial E_{k}}{\partial \dot{q}_{i}}\right)-\frac{\partial E_{k}}{\partial q_{i}}+\frac{\partial E_{p}}{\partial q_{i}}+\frac{\partial F}{\partial \dot{q}_{i}}=0, \\
& \vec{q}=\left[\begin{array}{llll}
x_{G} & z_{G} & \delta & \varepsilon
\end{array}\right]^{T} .
\end{aligned}
$$

Considering the pendulum system shown in Fig. 3, it is easy to see that the system has four degrees of freedom: three due to the hull (surge, heave and pitch motion) and the rotation of the pendulum around its hinge. These four variables can be used to describe the vector of generalized coordinates (see Eq. (3.3)).

The kinetic and potential energies are derived for both pendulum and the hull:

$$
\begin{aligned}
& E_{k, p}=\frac{1}{2} I_{y}(\dot{\delta}+\dot{\epsilon})^{2}+\frac{1}{2} m_{p}|\overrightarrow{O P}|^{2} \\
& E_{p, p}=m_{p} g(\overrightarrow{O P})_{z}=m_{p} g\left[z_{G}+d \cos \delta-l \cos (\delta+\epsilon)\right], \\
& E_{k, b}=\frac{1}{2} I_{b} \dot{\delta}^{2}+\frac{1}{2} m_{p}|\overrightarrow{O G}|^{2} \\
& E_{p, b}=m_{b} g(\overrightarrow{O G})_{z}=m_{b} g z_{G},
\end{aligned}
$$

where

$$
\begin{aligned}
& \overrightarrow{O P}=\left[\begin{array}{lll}
x_{G}+d \cdot \sin \delta-l \cdot \sin (\delta+\varepsilon) & 0 & z_{G}+d \cdot \cos \delta-l \cdot \cos (\delta+\varepsilon)
\end{array}\right]^{T}, \\
& \overrightarrow{O G}=\left[\begin{array}{lll}
x_{G} & 0 & z_{G}
\end{array}\right]^{T} .
\end{aligned}
$$


In this work, the torque control law presents an active (phased with the velocity) component, that ensure the extraction of energy. The PTO control law responds to the following equation:

$$
T_{\varepsilon}=c \dot{\varepsilon} .
$$

The corresponding Rayleigh dissipative function follows:

$$
F_{P T O}=\frac{1}{2} c \dot{\varepsilon}^{2} .
$$

It is important to observe that the expressions of the position and velocity vector used for the description of the kinetic and potential energy of pendulum and hull are expressed with respect to the $O-x y z$ coordinate system.

The procedure described above leads to the following differential equation:

$$
\mathbf{M}_{\mathbf{s}}\left[\begin{array}{c}
\ddot{x}_{G} \\
\ddot{z}_{G} \\
\ddot{\delta} \\
\ddot{\varepsilon}
\end{array}\right]+\mathbf{D}_{\text {PтO }}\left[\begin{array}{c}
\dot{x}_{G} \\
\dot{z}_{G} \\
\dot{\delta} \\
\dot{\varepsilon}
\end{array}\right]+F_{g r}+F_{\text {cor }}=0,
$$

where $\mathbf{M}_{\mathbf{S}}$ is the mass matrix of the system, $\mathbf{D}_{\mathbf{P T O}}$ is the damping matrix of the PTO.

$F_{\text {cor }}$ is the vector of Coriolis action due to the motion of the pendulum hinge and $F_{\mathrm{gr}}$ is the vector of the forces due to gravity action. The explicit expressions of these terms are as follows:

$$
\begin{aligned}
& \mathbf{M}_{\mathbf{s}}=\left[\begin{array}{cc}
m_{p}+m_{b} & 0 \\
0 & m_{p}+m_{b} \\
m_{p}[d \cos \delta-l \cos (\delta+\epsilon)] & -m_{p}[d \sin \delta-l \sin (\delta+\epsilon)] \\
-m_{p} l \cos (\delta+\epsilon) & m_{p} l \sin (\delta+\epsilon)
\end{array}\right. \\
& m_{p}[d \cos \delta-l \cos (\delta+\epsilon)] \quad-m_{p} l \cos (\delta+\epsilon) \\
& -m_{p}[d \sin \delta-l \sin (\delta+\epsilon)] \quad m_{p} l \sin (\delta+\epsilon) \\
& \left.\begin{array}{cc}
I_{b}+I_{y}+m_{p}\left(d^{2}+l^{2}\right)-2 m_{p} d l \cos \epsilon & I_{y}+m_{p} l^{2}-m_{p} d l \cos \epsilon \\
I_{y}+m_{p} l^{2}-m_{p} d l \cos \epsilon & I_{y}+m_{p} l^{2}
\end{array}\right], \\
& \mathbf{D}_{\text {Pто }}=\left[\begin{array}{llll}
0 & 0 & 0 & 0 \\
0 & 0 & 0 & 0 \\
0 & 0 & 0 & 0 \\
0 & 0 & 0 & c
\end{array}\right] \\
& F_{\mathrm{cor}}=\left[\begin{array}{c}
m_{p}\left[l(\dot{\delta}+\dot{\varepsilon})^{2} \sin (\delta+\varepsilon)-d \dot{\delta}^{2} \sin \delta\right] \\
m_{p}\left[l(\dot{\delta}+\dot{\varepsilon})^{2} \cos (\delta+\varepsilon)-d \dot{\delta}^{2} \cos \delta\right] \\
-m_{p} d l \sin \varepsilon\left[(\dot{\delta}+\dot{\varepsilon})^{2}-\dot{\delta}^{2}\right] \\
-m_{p} d l \dot{\delta}^{2} \sin \varepsilon
\end{array}\right],
\end{aligned}
$$




$$
F_{\mathrm{gr}}=\left[\begin{array}{c}
0 \\
0 \\
-m_{p} g[d \sin \delta-l \sin (\delta+\varepsilon)] \\
m_{p} g l \sin (\delta+\varepsilon)
\end{array}\right]
$$

The mass matrix $\mathbf{M}_{\mathbf{s}}$ can be rewritten as the sum of two contributions:

- The diagonal matrix $\mathbf{M}_{\mathrm{hp}}$ that takes into account the mass and inertial properties of the buoyant and of the pendulum;

$$
\mathbf{M}_{\mathrm{hp}}=\left[\begin{array}{cccc}
m_{p}+m_{b} & 0 & 0 & 0 \\
0 & m_{p}+m_{b} & 0 & 0 \\
0 & 0 & I_{b}+I_{y}+m_{p}\left(d^{2}+l^{2}\right)-2 m_{p} d l \cos \varepsilon & 0 \\
0 & 0 & 0 & I_{y}+m_{p} l^{2}
\end{array}\right] .
$$

- The matrix $\mathbf{M}_{\mathbf{c}}$ with nonnull off diagonal terms that takes into account the couplings between the pendulum and the hull.

$$
\mathbf{M}_{\mathbf{c}}=\left[\begin{array}{cc}
0 & 0 \\
0 & 0 \\
m_{p}[d \cos \delta-l \cos (\delta+\varepsilon)] & -m_{p}[d \sin \delta-l \sin (\delta+\varepsilon)] \\
-m_{p} l \cos (\delta+\varepsilon) & m_{p} l \sin (\delta+\varepsilon) \\
m_{p}[d \cos \delta-l \cos (\delta+\varepsilon)] & -m_{p} l \cos (\delta+\varepsilon) \\
-m_{p}[d \sin \delta-l \sin (\delta+\varepsilon)] & m_{p} l \sin (\delta+\varepsilon) \\
0 & I_{y}+m_{p} l^{2}-m_{p} d l \cos \varepsilon
\end{array}\right] .
$$

Then Eq. (3.12) may be rewritten as follows:

$$
\left(\mathbf{M}_{\mathbf{h p}}+\mathbf{M}_{\mathbf{c}}\right)\left[\begin{array}{c}
\ddot{x}_{G} \\
\ddot{z}_{G} \\
\ddot{\delta} \\
\ddot{\varepsilon}
\end{array}\right]+\mathbf{D}_{\mathbf{P T O}}\left[\begin{array}{c}
\dot{x}_{G} \\
\dot{z}_{G} \\
\dot{\delta} \\
\dot{\varepsilon}
\end{array}\right]+F_{\mathrm{gr}}+F_{\mathrm{cor}}=0 .
$$

\section{Hydrodynamic Model}

The dynamics of rigid bodies and fluid motion are governed by the combined actions of different external forces and moments. The forces and moment distributions and the kinematic description of the fluid motions are in fact continuous, assuming that the collection of discrete fluid molecules can be analyzed as a continuum [Journée and Massie, 2001]. 
However, in the case of a floating device, the motions of the hull can be evaluated through an integro-differential linear equation, called Cummins' equation [Cummins, 1962; Baghfalaki et al., 2012].

The coefficients of the equation are calculated via numeric tools such as ANSYS AQWA® or WAMITR [AQWA, 2013; WAMIT, 2015]; the calculation method is based on the linear potential flow theory and the panel method. The theory assumes that the flow is irrotational and inviscid and the fluid is incompressible. These assumptions are acceptable since the fluid velocities are low and the surface tension is negligible. Furthermore, the linear theory is accurate when the wave height is small with respect to the wavelength and the water depth [Airy, 1841].

The six DOFs equation of motion of a rigid floating marine structure with zero forward speed can be written in time domain under the assumption of linear phenomena, according to the decomposition reported in Eq. (4.2), developed by Ogilvie [1964].

$$
\begin{aligned}
& X=\left\{\begin{array}{c}
\text { surge } \\
\text { sway } \\
\text { heave } \\
\text { roll } \\
\text { pitch } \\
\text { yaw }
\end{array}\right\}=\left\{\begin{array}{c}
x \\
y \\
z \\
\operatorname{rot}_{x} \\
\operatorname{rot}_{y} \\
\operatorname{rot}_{z}
\end{array}\right\}, \\
& (\mathbf{M}+\mathbf{A}(\infty)) \ddot{X}+\int_{0}^{t} \mathbf{h}_{\mathbf{r}}\left(t-t^{\prime}\right) \dot{X} d t^{\prime}+\mathbf{K} X=\mathbf{F}_{\mathbf{w}}(t),
\end{aligned}
$$

where

- $\mathbf{M}$ is mass matrix of the floating body;

- $\mathbf{K}$ is the hydrostatic stiffness matrix;

- $\mathbf{A}(\infty)$ is the added mass matrix of the floating body, evaluated at infinite frequency;

- $\mathbf{h}_{\mathbf{r}}$ is denote impulse response functions of the radiation forces, that takes into account the fluid memory effect and incorporates the energy of the radiated waves generated by the motion of the body;

- $\mathbf{F}_{\mathbf{w}}(t)$ denote wave forces.

\section{Mooring Model}

Mooring system is modeled through a quasi-static approach. The system is mainly composed by three different lines (chains or lines) considered as rigid bodies with COG respectively in points $G_{1}, G_{2}, G_{3}$. A buoy (jumper) with a net buoyancy force $F_{b}$ is connected to point $A$ and a clump weight with net gravity force $F_{a}$ is connected to point $B$. The connection point of the mooring line with the device is point $C$. In Fig. 4, a scheme of the mooring line connected to the floater is provided. 
The static equilibrium of the system is studied varying the $x$ coordinate and the $z$ coordinate of the $C$ connection point in the mooring model reference frame. These variations represent all the different possible positions of the device compatibly with the maximum extension of the mooring line.

For all the different values of $x_{C \text {,Moor }}$ and $z_{C \text {,Moor }}$, the potential energy is calculated as a function of the angle $\theta_{1}$. The equilibrium condition is determined where the potential energy reaches its minimum value. The mooring line tension $F_{m \text {,Moor }}$ at the connection point $C$ can be calculated respect to the mooring reference frame. Tension is decomposed along the $x_{\text {Moor }}$ and $z_{\text {Moor }}$ coordinates.

The action of the waves on the hull induces the surge, heave and pitch motions, which are computed with respect to the $O$-xyz e reference frame.

In order to obtain the mooring tensions (which are expressed in the mooring reference frame), it is necessary to evaluate the motion of point $C$ with respect mooring reference frame $M-x_{\text {Moor }} y_{\text {Moor }} z_{\text {Moor }}$.

The motion of point $C$ with respect to the mooring coordinate system can be computed starting from hull motions expressed in the $O$-xyz coordinate system, thanks to a transformation matrix between the two coordinate systems considered.

The position of point $C$ with respect to $G-x_{1} y_{1} z_{1}$ coordinate system is

$$
{ }^{1} \vec{p}_{C}=\left[\begin{array}{c}
x_{C} \\
0 \\
z_{C}
\end{array}\right] .
$$

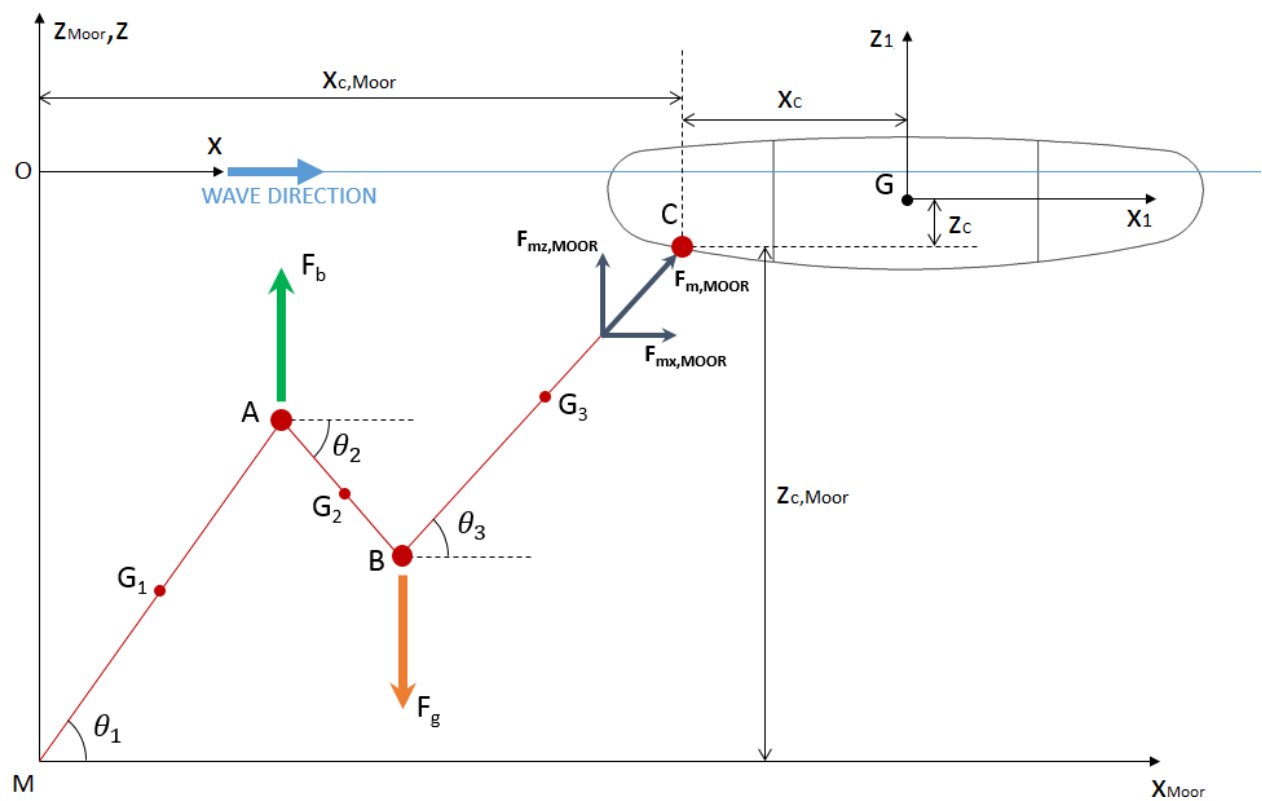

Fig. 4. Mooring model layout. 
The apex of the notation ${ }^{1} \vec{p}_{C}$ indicates the reference frame considered in order to express the position indicated by the subscript.

It is noticeable that the value of $x_{C}$ is negative, while $z_{C}$ can be both positive or negative, according to its position with respect to the hull COG.

The position of hull COG with respect to mooring coordinate system is

$$
{ }^{\text {Moor }} \vec{p}_{G}=\left[\begin{array}{c}
x_{C} \\
0 \\
H_{0 G}+z_{G}
\end{array}\right]=\left[\begin{array}{c}
x_{C} \\
0 \\
\left(z_{C, \text { Moor }}+\left|z_{C}\right|\right)+z_{G}
\end{array}\right] \text {. }
$$

The homogeneous transformation matrix that allows to express, in the mooring reference frame, the orientation and the position of a vector described in the $O-x y z$ coordinate system is

$$
\text { Moor } \hat{\mathbf{A}}_{\mathbf{1}}=\left[\begin{array}{cccc}
\cos \delta & 0 & \sin \delta & x_{C} \\
0 & 1 & 0 & 0 \\
-\sin \delta & 0 & \cos \delta & H_{0 G}+z_{G} \\
0 & 0 & 0 & 1
\end{array}\right] .
$$

The position of point $C$ in mooring coordinate system can be defined in the following way:

$$
{ }^{\text {Moor }} \overrightarrow{\hat{p}}_{C}={ }^{\text {Moor }} \hat{\mathbf{A}}_{\mathbf{1}} \cdot{ }^{1} \overrightarrow{\hat{p}}_{C}=\left[\begin{array}{c}
x_{C}+x_{C} \cos \delta+z_{C} \sin \delta \\
0 \\
\left(H_{0 G}+z_{G}\right)-x_{C} \sin \delta+z_{C} \cos \delta \\
1
\end{array}\right] .
$$

Using the hull motions computed in the $O$-xyz reference frame, it is possible to evaluate the position of point $C$ in $M-x_{\text {Moor }} y_{\text {Moor }} z_{\text {Moor }}$ reference frame and then the forces due to the mooring lines, computed in the same coordinate system.

At this point, the moor tension evaluated through two Matlab look-up tables can be transported in the $O$-xyz reference frame thanks to a transformation matrix. Three different contribution can be distinguished:

- ${ }^{1} F_{m x}$ : mooring force along the $x$-axis;

- ${ }^{1} F_{m z}$ : mooring force along the $z$-axis;

- ${ }^{1} F_{m r y}$ : mooring moment about the $y$-axis.

The mooring line forces discharged on the hull, along $x$ and $z$ axis, have the same modulus of the ones computed with look-up table (LUT), but with opposite direction:

$$
\begin{aligned}
& { }^{1} F_{m x}=-\left({ }^{\text {Moor }} F_{m x}\right) \\
& { }^{1} F_{m z}=-\left({ }^{\text {Moor }} F_{m z}\right) .
\end{aligned}
$$


In the end, the torque discharged on the hull can be computed considering the static condition of the hull:

$$
\begin{aligned}
{ }^{1} F_{m r y}= & -\sqrt{x_{C}^{2}+z_{C}^{2}}\left[-\left({ }^{\text {Moor }} F_{m x} \sin \left(\tan ^{-1}\left(\frac{\left|z_{C}\right|}{\left|x_{C}\right|}\right)-\delta\right)\right)\right. \\
& \left.+\left({ }^{\text {Moor }} F_{m z} \cos \left(\tan ^{-1}\left(\frac{\left|z_{C}\right|}{\left|x_{C}\right|}\right)-\delta\right)\right)\right] .
\end{aligned}
$$

\section{Full Model}

The full model is obtained combining the nonlinear pendulum dynamics equation with the hydrodynamic ones. The vector $X$ of the hydrodynamic model was reduced neglecting the sway, roll and yaw degrees of freedom: the hydrodynamic model can then be coupled with planar representation of the mechanics.

$$
X=\left\{\begin{array}{l}
x \\
z \\
\delta \\
y
\end{array}\right\} .
$$

Furthermore, it is necessary to add the following elements:

- $F_{\text {drift }}$ : mean drift force along surge direction, due to the action of the waves on the hull;

- $F_{m}$ : mooring force estimated via quasi-static model described in the previous section;

- $F_{\mathrm{PTO}}$ : force exerted by the PTO.

Mean drift force is required in order to complete properly the hydrodynamic model along the surge direction. It is important to higlight that the motion of the pendulum is strongly coupled with surge motion. Considering that the hydrodynamic coefficients are obtained through a potential flow code, only the mean drift force is taken into account, since it does not depend on the second order terms of the velocity potential [Pinkster, 1980; Faltinsen, 1990; Prins, 1995; Huijsmans, 1996]. On the other hand, considering this degree of freedom, it is necessary to include the mooring force exercited in order to keep in place the device.

Considering the matrix representation, we have

$$
\begin{aligned}
\left(\mathbf{M}_{\mathbf{s}}\right. & +\mathbf{A}(\infty)) \ddot{X}+\int_{0}^{t} \mathbf{h}_{\mathbf{r}}\left(t-t^{\prime}\right) \dot{X} d t^{\prime}+\mathbf{K} X \\
& =F_{\mathrm{drift}}+F_{w}+F_{\mathrm{cor}}+F_{\mathrm{gr}}+F_{m}+F_{\mathrm{PTO}} .
\end{aligned}
$$

The time domain equation of the full model, described above, was implemented through a Matlab/Simulink® numerical model. In Fig. 5, the main level of the numerical model is represented. 


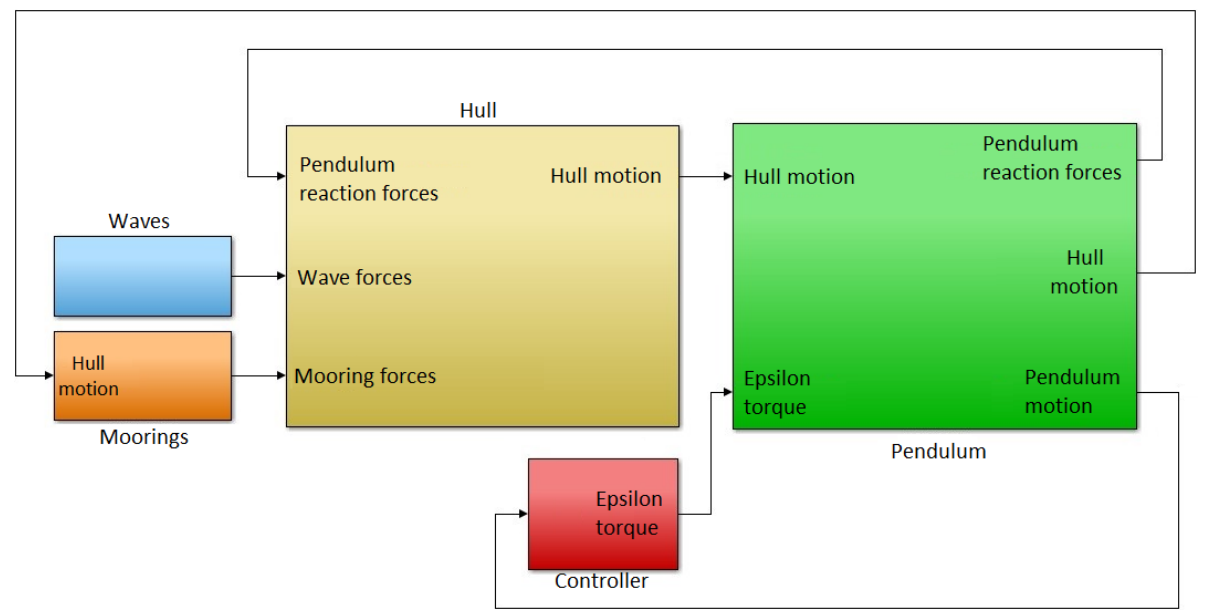

Fig. 5. Matlab/Simulink® model main level layout.

The model is composed of five main blocks:

- WAVES: Input block, containing the mean drift force along surge axis and the wave forcing functions for surge, heave and pitch directions;

- HULL: In this block, Cummins equation is implemented. The outputs are the hull motion along surge, heave and pitch directions;

- PENDULUM: Given the motion of the hull, this block computes the angular displacement, velocity and acceleration of the pendulum. Furthermore, the forward dynamic is also computed, so it is possible to determine the reaction forces of the pendulum. These forces are given as feedback to the hull;

- MOOR: Mooring line behavior is described through a quasi-static model; in this block mooring forces are computed using LUT, which contains the characteristic force-displacement of the mooring line. The force computed is the third input for the HULL block;

- CONTROLLER: Here the control law is implemented, taking into account also the limits of the PTO, such as maximum torque and power.

\section{Simplified Extracted Power Equation}

As mentioned before, the research activity on the PeWEC device is focused on the design of a pendulum converter for the Mediterranean Sea, where waves are shorter, thus with a higher frequency.

Therefore, the aim of this section is to show that the extracted power is somehow proportional to the frequency of the incoming wave.

This characteristic can be demonstrated starting from the four degrees nonlinear model presented in Sec. 3.2 and applying some simplifications, in order to decouple the surge, pitch and pendulum degrees of freedom. 
It is important to highlight, since the beginning, that the simplified model here presented is only a mathematical justification about the focus on the design of a pendulum converter for a Mediterranean site. In fact, this model will not be used for the computation of the numerical results presented in Chap. 9.

\subsection{Linear mechanical equations}

The equations obtained before with the Lagrange approach are a set of nonlinear equations. In general, in the initial design phase, it is quite interesting working with a linear system, because it allows using different mathematical tools useful for the system's comprehension and development.

The main hypothesis for the linearization of the equations is to assume that the pitch angle and the angular rotation of the pendulum are small enough and that the system oscillate around the equilibrium position defined for $\varepsilon=0, \delta=0$.

Then under these assumptions, the equations of motion become

$$
\left(\mathbf{M}_{\mathbf{h p}}^{\operatorname{lin}}+\mathbf{M}_{\mathbf{c}}^{\mathbf{l i n}}\right)\left[\begin{array}{c}
\ddot{x}_{G} \\
\ddot{z}_{G} \\
\ddot{\delta} \\
\ddot{\varepsilon}
\end{array}\right]+\mathbf{D}_{\mathbf{P T O}}\left[\begin{array}{c}
\dot{x}_{G} \\
\dot{z}_{G} \\
\dot{\delta} \\
\dot{\varepsilon}
\end{array}\right]+\mathbf{K}_{\mathbf{p}}\left[\begin{array}{c}
x_{G} \\
z_{G} \\
\delta \\
\varepsilon
\end{array}\right]=0 .
$$

$\mathbf{K}_{\mathbf{p}}$ is the restoring matrix of the system and depends on the linearized terms of the gravitational vector, $\mathbf{M}_{\mathbf{h p}}^{\mathrm{lin}}$ and $\mathbf{M}_{\mathbf{c}}^{\mathrm{lin}}$ are the linearized matrix of the inertial actions of the system. The Coriolis vector is negligible, since it depends on the square of the angular pitching velocity and pendulum angular velocity.

$$
\begin{aligned}
\mathbf{K}_{\mathbf{p}}= & {\left[\begin{array}{cccc}
0 & 0 & 0 & 0 \\
0 & 0 & 0 & 0 \\
0 & 0 & -m_{p} g(d-l) & m_{p} g l \\
0 & 0 & m_{p} g l & m_{p} g l
\end{array}\right], } \\
\mathbf{M}_{\mathbf{h p}}^{\operatorname{lin}}= & {\left[\begin{array}{cccc}
m_{p}+m_{b} & 0 & 0 & 0 \\
0 & m_{p}+m_{b} & 0 & 0 \\
0 & 0 & I_{y}+I_{b}+m_{p}(d-l)^{2} & 0 \\
0 & 0 & 0 & I_{y}+m_{p} l^{2}
\end{array}\right], } \\
\mathbf{M}_{\mathbf{c}}^{\operatorname{lin}}= & {\left[\begin{array}{cccc}
0 \\
0 & 0 & m_{p}(d-l) & 0 \\
m_{p}(d-l) & 0 & 0 & I_{y}+m_{p} l^{2}-m_{p} d l \\
-m_{p} l & 0 & I_{y}+m_{p} l^{2}-m_{p} d l & 0
\end{array}\right] . }
\end{aligned}
$$




\subsection{Simplified PeWEC dynamics}

The linear mechanical equations described in Sec. 7.1 can be coupled with the hydrodynamic model presented in Chapter 4 . Then the model can be further simplified, neglecting the couplings between the surge, pitch and pendulum degrees of freedom. This simplification is based on the following hypothesis:

- The hull is retained with a low-stiffness mooring line: this implies that the surge motion response has a period greater than the wave period of the incoming wave. Then the floating structure responds poorly to the first-order wave forces acting along the surge direction. Such forces are the ones that most contribute to the excitation of the pendulum. Taking into account these considerations, the coupling between surge — pitch and surge — pendulum coordinate can be neglected;

- The wave is supposed to be monochromatic, so the convolution integral for pitch motion can be substituted with the frequency dependent hydrodynamic damping coefficient $B_{55}(\omega)$;

- Considering again the approximation of the convolution integral, also the added mass evaluated for infinite frequency can be replaced with the frequency dependent added mass coefficient $A_{55}(\omega)$;

- The heave motion can be neglected, since it is uncoupled with the other degrees of freedom.

Applying such hypothesis, the system of equations (7.1), completed with the hydrodynamic terms of Eq. (4.2), becomes

$$
\left\{\begin{array}{c}
{\left[I_{b}+I_{y}+m_{p}(d-l)^{2}+A_{55}(\omega)\right] \ddot{\delta}+B_{55}(\omega) \dot{\delta}+\left[K_{55}-m_{p} g l\right] \delta} \\
\quad=F_{w, 5}(\omega)-\left(I_{y}+m_{p} l^{2}-m_{p} d l\right) \ddot{\varepsilon}-m_{p} g l \varepsilon \\
\left(I_{y}+m_{p} l^{2}\right) \ddot{\varepsilon}+c \dot{\varepsilon}+m_{p} g l \varepsilon=-\left(I_{y}+m_{p} l^{2}-m_{p} d l\right) \ddot{\delta}-m_{p} g l \delta .
\end{array}\right.
$$

By giving to the system a sinusoidal input $\delta=\frac{\delta_{0}}{2}\left(e^{j \omega t}+e^{-j \omega t}\right)$ and expecting a sinusoidal response $\varepsilon=\frac{\varepsilon_{0}}{2}\left(e^{j(\omega t+\vartheta)}+e^{-j(\omega t+\vartheta)}\right)$, the amplitude $\varepsilon_{0}$ can be linked to $\delta_{0}$ through the following transfer function:

$$
H_{\varepsilon \delta}(j \omega)=\frac{\varepsilon_{0}}{\delta_{0}}=\frac{\left[\left(I_{y}+m_{p} l^{2}-m_{p} d l\right) \omega^{2}-m_{p} g l\right]}{\left[-\omega^{2}\left(I_{y}+m_{p} l^{2}\right)+j \omega c+m_{p} g l\right]} e^{j \vartheta} .
$$

The extracted power from the PTO can be expressed as the product between the angular velocity of the pendulum and the torque at its shaft. The torque at the PTO shaft responds to the control law given by Eq. (3.10). Deriving the $\varepsilon$ expression with respect to the time, the equation of the extracted power result will be

$$
P_{\varepsilon}=T_{\varepsilon} \dot{\varepsilon}=c \dot{\varepsilon}^{2}=c \frac{\varepsilon_{0}^{2}}{4} \omega\left(2-e^{2 j(\omega t+\theta)}-e^{-2 j(\omega t+\theta)}\right) .
$$

The average extracted power from the PTO, on the other hand, is governed by the following equation:

$$
P_{\varepsilon, \mathrm{avg}}=\frac{1}{T} \int_{0}^{T} P_{\varepsilon} d t=\frac{1}{2} \omega^{2} c \varepsilon_{0}^{2}=\frac{1}{2} \omega^{2} c\left|H_{\varepsilon \delta}(j \omega)\right|^{2} \delta_{0}^{2} .
$$


Substituting Eq. (7.6) in Eq. (7.8) and considering the resonance frequency of the pendulum, we have

$$
\omega_{p}=\sqrt{\frac{m_{p} g l}{\left(I_{y}+m_{p} l^{2}\right)}},
$$

the average extracted power formula results in

$$
P_{\varepsilon, \text { avg }}=\frac{\omega^{2} c}{2} \frac{\left(I_{y}+m_{p} l^{2}\right)^{2}\left[\left(1-\omega_{p}^{2} \frac{d}{g}\right) \omega^{2}-\omega_{p}^{2}\right]^{2}}{\left(I_{y}+m_{p} l^{2}\right)^{2}\left(\omega_{p}^{2}-\omega^{2}\right)^{2}+\omega^{2} c^{2}} \delta_{0}^{2} .
$$

Equation (7.10) highlights that the maximum power is absorbed when the system is resonating [Bracco et al., 2011]. In resonance conditions, the power can be expressed as follows:

$$
P_{\varepsilon, \mathrm{res}}=\frac{\left(I_{y}+m_{p} l^{2}\right)^{2}\left[\left(-\omega_{p}^{2} \frac{d}{g}\right) \omega^{2}\right]^{2}}{2 c} \delta_{0}^{2} .
$$

In resonance conditions, the damping coefficient can be evaluated through Eq. (7.8):

$$
c=\frac{2 P_{\varepsilon, \text { res }}}{\omega^{2} \varepsilon_{0}^{2}} .
$$

Substituting Eq. (7.12) into Eq. (7.10) and recalling the pendulum resonance frequency given by Eq. (7.9), the equation of the average extracted power becomes

$$
P_{\varepsilon, \text { avg }}=\frac{1}{2}\left(m_{p} l d\right) \omega^{3} \delta_{o} \varepsilon_{0} .
$$

Equation (7.13) shows that, considering the simplified model, the average absorbed power is proportional to the pendulum mass $m_{p}$ and length $l$, the distance between the pendulum hinge and the hull COG, the cube of the wave frequency $\omega$, the amplitude of pitch motion $\delta_{0}$ and the amplitude of oscillation of the PTO shaft $\varepsilon_{0}$.

\section{1:12 Scale Prototype}

During the research activity at the Politecnico di Torino, the 1:12 scaled prototype shown in Fig. 6 was designed and built. The prototype was designed considering the wave scatter diagram of Pantelleria, the site where the full scale ISWEC prototype was installed in July 2014. The design point was the most energetic wave, its significant height and energy period are reported in Table 1. Then, through the iso-energetic approach, the equivalent regular wave was considered and the scale of the PeWEC prototype was chosen taking into account the INSEAN wave basin (Rome) specifications [INSEAN, 2001].

Given the wave properties shown in Table 1, several simulations were performed in order to find the shape of the hull. The following design criteria to maximize the hull performances are chosen:

- The natural frequency of the hull as close as possible to the frequency of the chosen wave; 


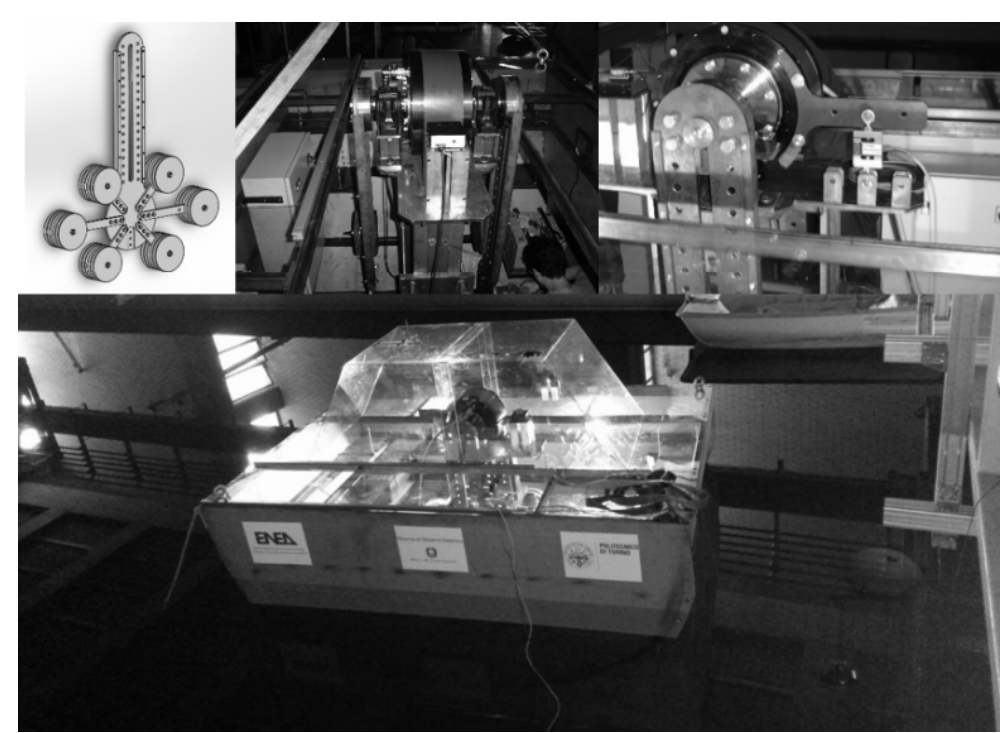

Fig. 6. Pendulum on the left, PTO and PTO frame in the middle, PTO load cell detail on the right and moored device at the INSEAN wave tank on the bottom.

Table 1. Most energetic wave in Pantelleria Island site and 1:12 scaled regular wave properties.

\begin{tabular}{|c|c|c|}
\hline$\overline{\text { Symbol }}$ & Description & Value \\
\hline \multicolumn{3}{|c|}{ Most energetic wave in Pantelleria Island site } \\
\hline$\overline{\mathrm{H}_{\mathrm{S}}}$ & Significant wave height & $2.65 \mathrm{~m}$ \\
\hline $\mathrm{T}_{\mathrm{e}}$ & Energy period & $7.75 \mathrm{~s}$ \\
\hline $\mathrm{P}_{\mathrm{w}, \text { irr }}$ & Power density & $26.67 \mathrm{~kW} / \mathrm{m}$ \\
\hline \multicolumn{3}{|c|}{ 1:12 scaled regular wave } \\
\hline$\overline{\mathrm{H}}$ & Wave height & $0.15 \mathrm{~m}$ \\
\hline $\mathrm{T}$ & Wave period & $2.2 \mathrm{~s}$ \\
\hline $\mathrm{P}_{\mathrm{w}, \mathrm{reg}}$ & Power density & $50 \mathrm{~W} / \mathrm{m}$ \\
\hline
\end{tabular}

- Length of the floating body between one third of and half of the wavelength;

- Minimal added mass and dissipation.

The first criterion is based on the principle that, in regular waves, energy is exploited most efficiently when the un-damped natural frequency of the device is close to the dominant frequency of the incoming wave. The second criterion is a compromise between using the maximum slope of the waves and reducing the floater size and costs, maximizing the performances of the system.

The third criterion was adopted to minimize energy dissipation and ineffective interactions between waves and hull. Furthermore, in order to avoid hydrodynamic interactions between the buoy and the tank walls, the width of the hull was chosen to be smaller than one quarter of the tank width. 
The optimization procedure led to the cylindrical shape as the best shape. Considering the criteria described above, the hull was $3 \mathrm{~m}$ long and $2 \mathrm{~m}$ wide; as consequence the radius of the cylindrical section was $1.5 \mathrm{~m}$. The hull inertia and displacement were adjusted in order to achieve the design wave period and the hydrostatic stability.

The PTO was suspended in the middle of the hull width, through a motorized frame fixed on a reinforced steel structure welded on the bottom of the hull. The frame was designed in order to allow the variation of the vertical position of the pendulum hinge with respect to the hull center of gravity, or rather to adjust the distance $d$.

In order to maintain the symmetry of the device, one pendulum was positioned at each extremity of the PTO shaft. Each pendulum was built with a central steel structure and six steel bars used for the suspension of some steel discs. In this way, it was possible to change the pendulum mass and inertia. Furthermore, on the central steel structure, a series of holes were provided in order to change the pendulum length. These adjustments were useful for the pendulum resonance tuning and the analysis of the pendulum mass influence on the behavior of the device.

The PTO was a permanent magnet motor directly coupled with the pendulums. The angular displacement and velocity of the pendulums were measured thanks to the encoder integrated into the electrical machine. The torque at the PTO shaft was

Table 2. Feature of the main configuration prototype.

\begin{tabular}{|c|c|c|}
\hline$\overline{\text { Symbol }}$ & Description & Value \\
\hline \multicolumn{3}{|c|}{ Hull } \\
\hline$\overline{\mathrm{L}}$ & Length & $3 \mathrm{~m}$ \\
\hline $\mathrm{R}$ & Radius & $1.5 \mathrm{~m}$ \\
\hline W & Width & $2 \mathrm{~m}$ \\
\hline $\mathrm{m}_{b}$ & Mass & $3176 \mathrm{~kg}$ \\
\hline & Roll axis moment of inertia & $1499 \mathrm{kgm}^{2}$ \\
\hline $\mathrm{I}_{\mathrm{yy}}=\mathrm{I}_{\mathrm{b}}$ & Pitch axis moment of inertia & $2168 \mathrm{kgm}^{2}$ \\
\hline & Yaw axis moment of inertia & $2761 \mathrm{kgm}^{2}$ \\
\hline $\mathrm{d}$ & Pendulum hinge - hull COG distance & $0.858 \mathrm{~m}$ \\
\hline \multicolumn{3}{|c|}{ Pendulum } \\
\hline$\overline{m_{p}}$ & Mass & $410 \mathrm{~kg}$ \\
\hline $\mathrm{I}_{\mathrm{y}}$ & Moment of inertia with respect to $\mathrm{COG}$ & $88.18 \mathrm{kgm}^{2}$ \\
\hline 1 & Length & $0.866 \mathrm{~m}$ \\
\hline \multicolumn{3}{|c|}{ PTO } \\
\hline$\overline{\mathrm{T}_{\text {nom }}}$ & Rated torque & $220 \mathrm{Nm}$ \\
\hline $\mathrm{T}_{\max }$ & Maximum torque & $800 \mathrm{Nm}$ \\
\hline $\mathrm{V}_{\mathrm{n}}$ & Rated speed & $90 \mathrm{rpm}$ \\
\hline \multicolumn{3}{|c|}{ Mooring } \\
\hline$\overline{\mathrm{F}_{\mathrm{g}}}$ & Net gravity force & $98 \mathrm{~N}$ \\
\hline $\mathrm{F}_{\mathrm{b}}$ & Net buoyancy force & $250 \mathrm{~N}$ \\
\hline$l_{1}$ & Line 1 length & $1.8 \mathrm{~m}$ \\
\hline$l_{2}$ & Line 2 length & $0.4 \mathrm{~m}$ \\
\hline$l_{3}$ & Line 3 length & $2 \mathrm{~m}$ \\
\hline
\end{tabular}




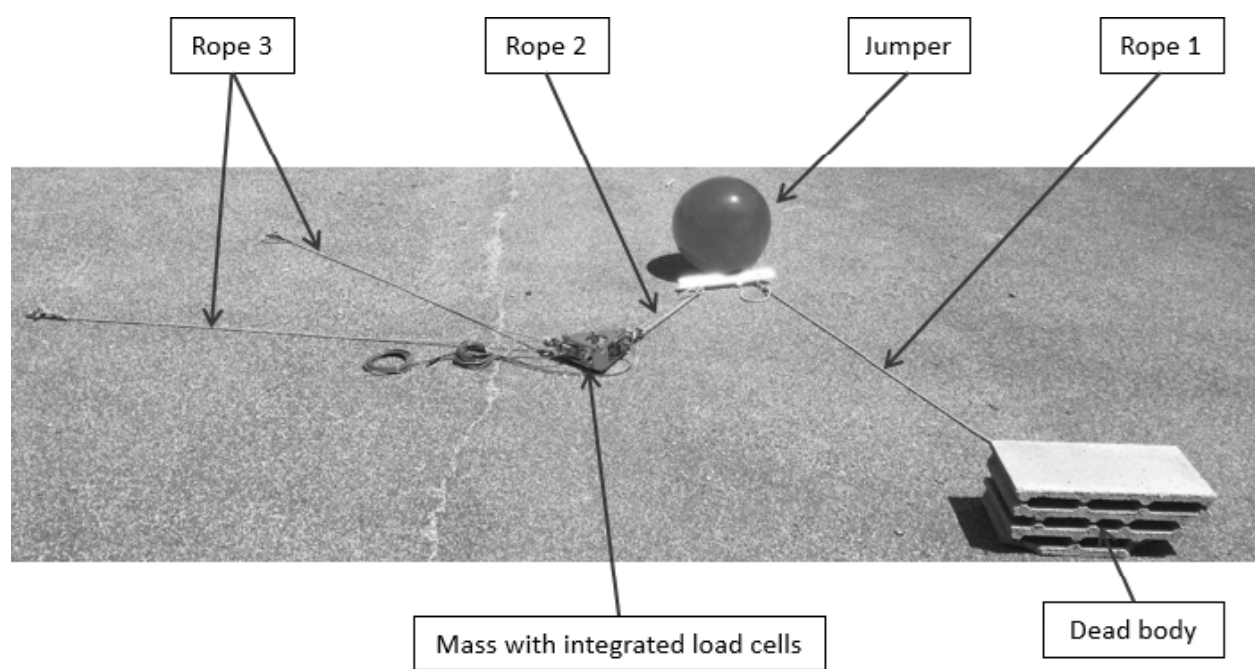

Fig. 7. Mooring line arrangement during installing operations.

measured through the load cell connected to the PTO stator, as shown in Fig. 6 . Table 2 summarizes the prototype characteristics of the main configuration.

The compliant mooring line was constituted by a submerged jumper and a mass connected, from one side to the seabed and to the other side to the hull, through three lines.

The behavior of this kind of mooring is comparable to a hardening spring behavior with reduced stiffness for small displacement and high restoring force at big displacements.

Such behavior is desired because it allows small forces on the PeWEC for normal operations and high forces before end stroke, thus reducing snatches in extreme wave conditions. In Fig. 7, the mooring arrangement during the installing operation is shown.

The jumper was realized with submerged buoy with $250 \mathrm{~N}$ of net buoyancy, while the $98 \mathrm{~N}$ mass was constituted by a structure designed to house two submergible load cells, used for the measuring of lines 2 and 3 tensions. Line 3 finishes with two arms connected to the left and right side of the hull to help the self-alignment of the device with respect to the wave.

\section{Numerical System Response}

In this section, the influence of the control damping parameter and of the wave period on the behavior of the PeWEC device is analyzed. The aim of this section is to understand the system dynamics, considering the nonlinear pendulum equations coupled with the linear hydrodynamics and taking into account the main configuration parameters of the 1:12 scaled prototype given in Table 2. 

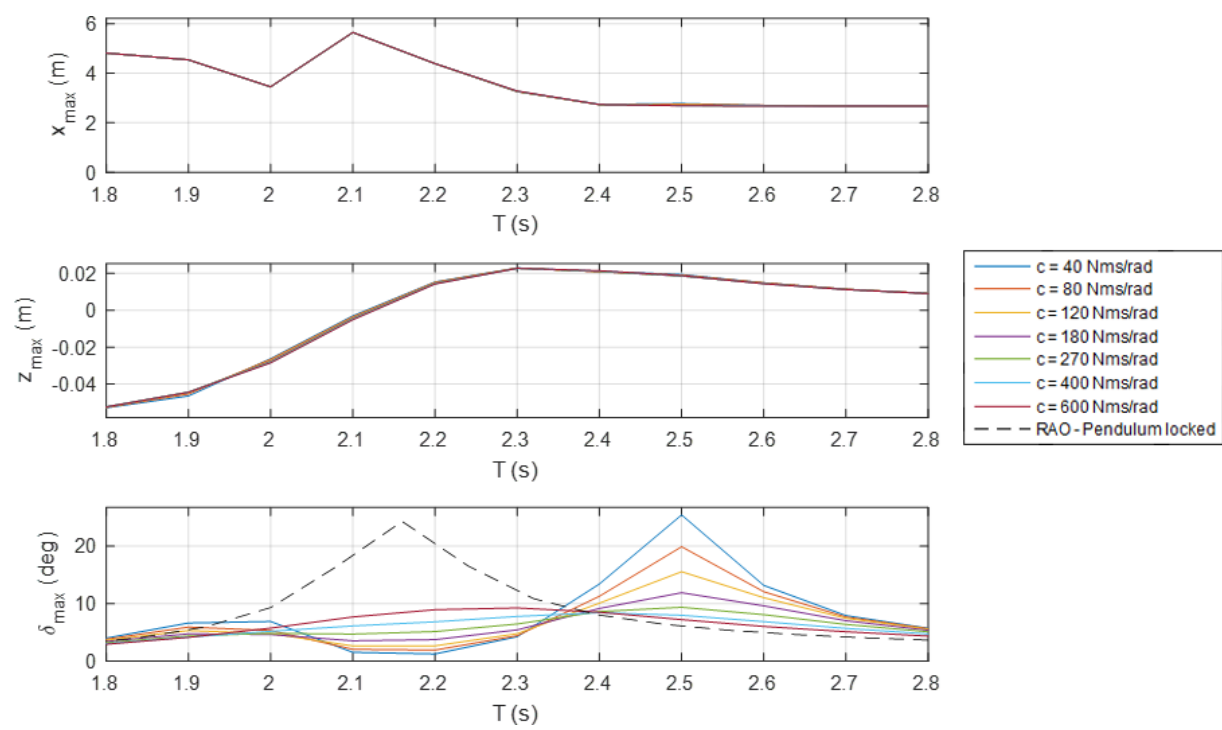

Fig. 8. (Color online) Overall system: hull response in the surge, heave and pitch degrees of freedom, for different values of the PTO damping coefficient (colored lines) and pitch RAO with locked pendulum (dashed black line).

The excitation of the system is a regular wave with period varying from $1.8 \mathrm{~s}$ and $2.8 \mathrm{~s}$ and height equal to $0.15 \mathrm{~m}$ (the height of the design scaled regular wave). On the other hand, the control damping parameter varies between $40 \mathrm{Nms} / \mathrm{rad}$ and $600 \mathrm{Nms} / \mathrm{rad}$.

Figures 8 and 9 show, the hull motion response and the velocity amplitude along surge, heave and pitch directions, respectively.

The amplitude of the motion response along surge and heave axis is not influenced by the control damping coefficient but only from the wave period of the incoming wave. On the other hand, the velocity response along surge axis shows a dependency from the control parameter.

A horizontal asymptote for longest wave periods can be identified both for the response along surge and heave axis. This is due to the fact that for very long waves the floating body tends to follow the wave crests and through.

The motion and the velocity response along pitch direction are influenced by the control law coefficient. In particular, it is noticeable that, in correspondence of the resonant period of the hull $(2.2 \mathrm{~s})$, the coupling of the hull and the pendulum determines a damping of the pitch motion. The degree of damping decrease increasing the PTO control law parameter. Indeed, if an infinite value of the PTO damping coefficient is considered, then the pitch response of the coupled system tends to the one obtained with the locked pendulum (dashed black line in Fig. 8).

Greater amplitudes of pitch motion can be observed in correspondence of the $2.5 \mathrm{~s}$ wave period, when damping coefficient is set between $40 \mathrm{Nms} / \mathrm{rad}$ and $120 \mathrm{Nms} / \mathrm{rad}$. 

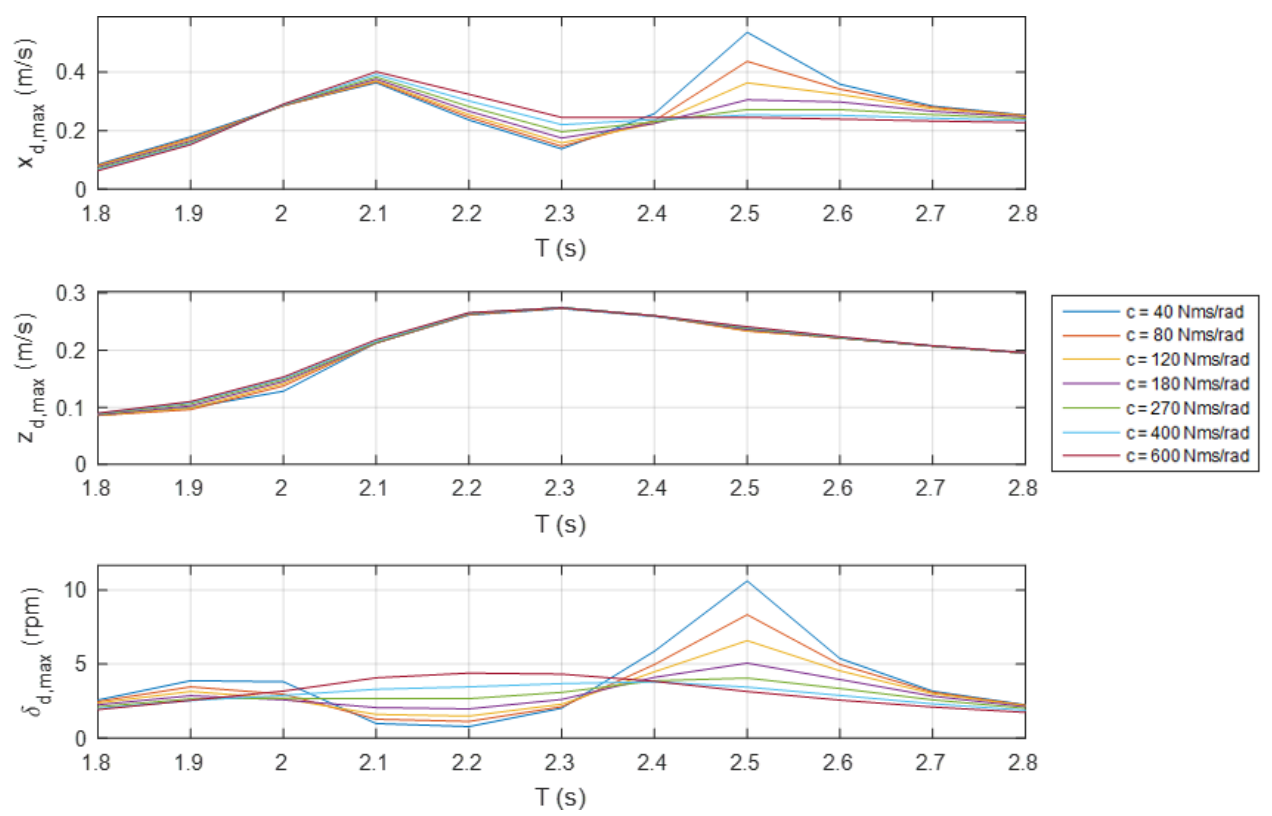

Fig. 9. (Color online) Overall system: hull velocity amplitude in the surge, heave and pitch degrees of freedom, for different values of the PTO damping coefficient.

The same observations may be applied for what concern the pitch velocity response.

Figure 10 shows the pendulum response and the PTO torque and extracted power. For relatively small PTO damping coefficients (between $40 \mathrm{Nms} / \mathrm{rad}$ and $120 \mathrm{Nms} / \mathrm{rad}$ ), a peak can be found at 2.5 wave period, where the pitch amplitude reaches its maximum values too. Between $1.9 \mathrm{~s}$ and $2.2 \mathrm{~s}$, a sort of plateau can be observed despite the pitch response tends to have a minimum around $2.2 \mathrm{~s}$. The origin of the plateau can be found considering the fact that the pendulum motion is influenced both from surge and pitch motion. Indeed, in the wave period interval mentioned above, the surge motion reaches its maximum amplitude and here its contribution helps the excitation of the pendulum.

Increasing the control damping coefficient, the peak at $2.5 \mathrm{~s}$ becomes smoothed and in the case of its highest values the pendulum becomes almost constant, with a maximum around the design wave period.

Since the torque is proportional to the pendulum angular velocity by the PTO damping coefficient, the behavior of the torque characteristic shown Fig. 10 is quite similar to the one of the pendulum angular velocity. In particular, the torque increases increasing the damping coefficient and the peak at $2.5 \mathrm{~s}$, that can be seen for small values of the control parameters, moves back at the wave period of $2.2 \mathrm{~s}$.

By varying properly the PTO control parameter, we can highlight a range of wave period in which the power extraction is maximized. In the case of the prototype 

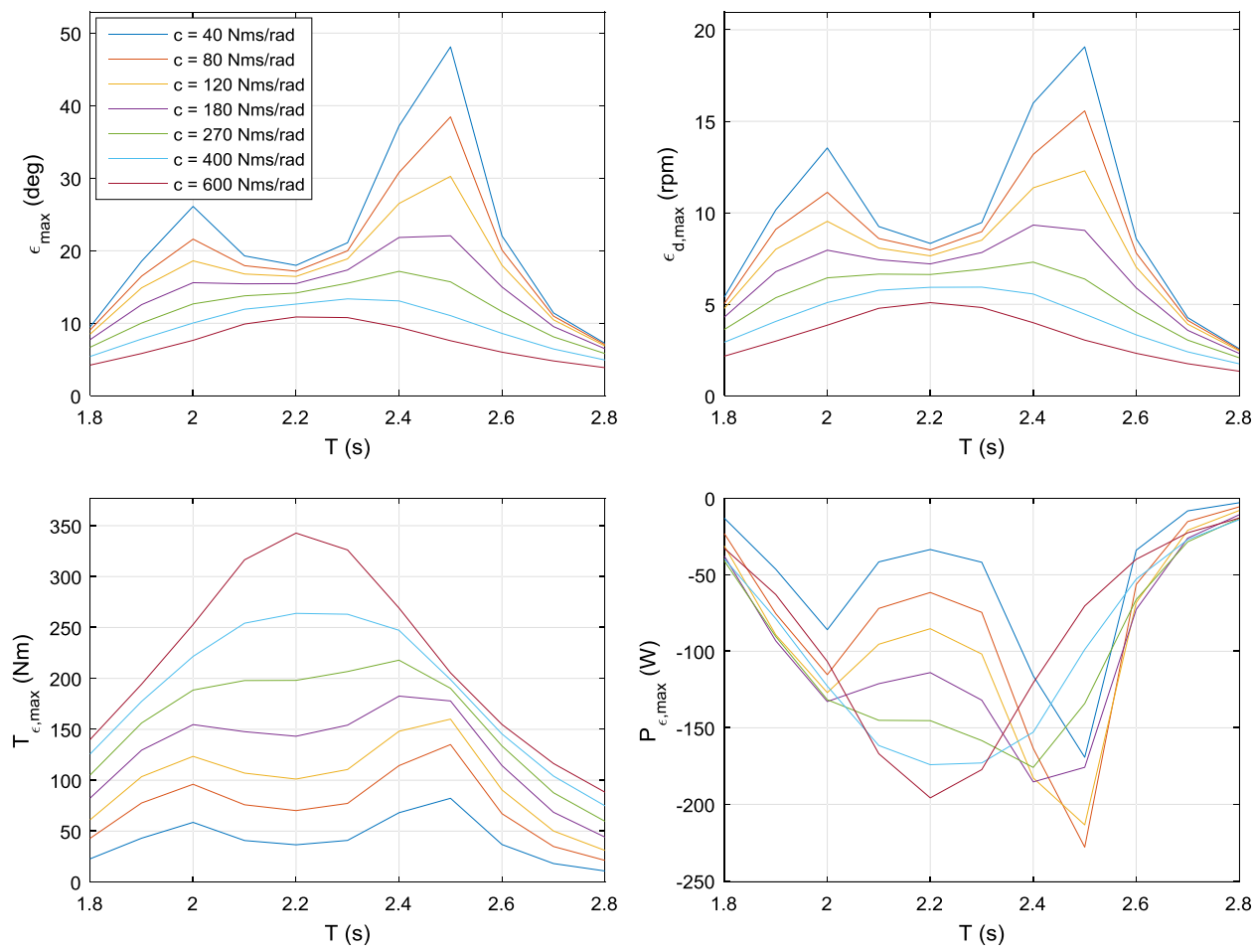

Fig. 10. Overall system: pendulum position and speed, PTO torque and power.

considered, from $2 \mathrm{~s}$ to $2.3 \mathrm{~s}$ wave period the maximum extracted power can be maximized between $105 \mathrm{~W}$ and $140 \mathrm{~W}$; then at $2.5 \mathrm{~s}$ the maximum extracted power can reach the absolute maximum of $235 \mathrm{~W}$. After this peak, the power decreases rapidly. This result is interesting since the performances of the device are noticeable for a quite wide range wave periods.

\section{Experimental and Numerical Comparison}

In this section, the numerical and experimental comparison is presented with the aim to evaluate the accuracy of the numerical model developed in Chapters 4, 5 and 6 with respect to the real behavior of the prototype. In particular, the analysis is focused on the evaluation of the relative error of the model near the resonant condition of the WEC.

\subsection{Frequency sweep}

The first result shown is the comparison between the numerical and the experimental frequency sweep obtained with a constant value of the PTO damping coefficient. In particular, this parameter was set at $40 \mathrm{Nms} / \mathrm{rad}$, while the wave period was 

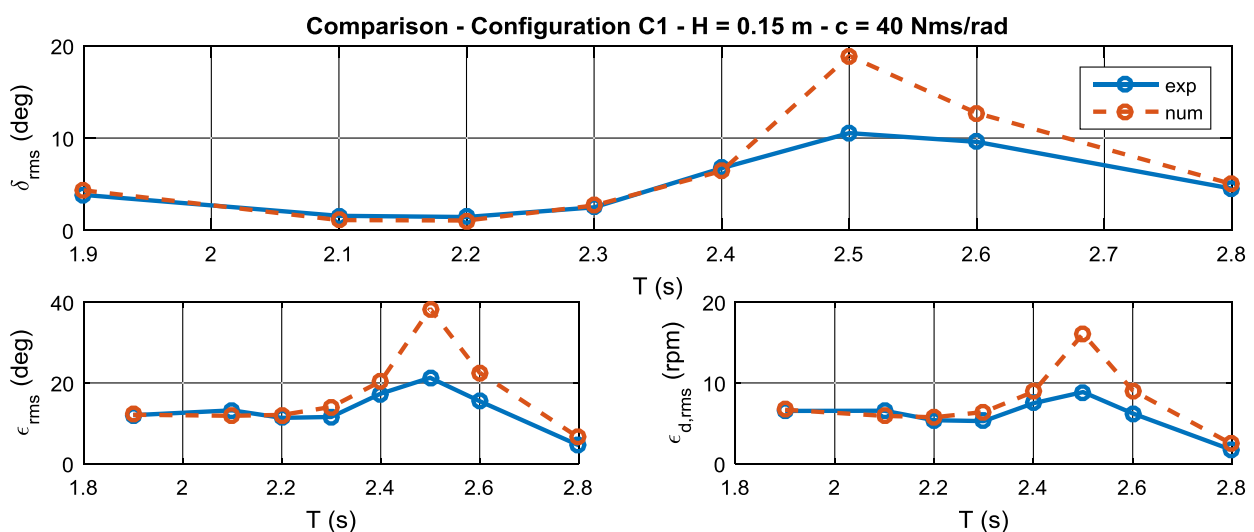

$\mathrm{T}(\mathrm{s})$
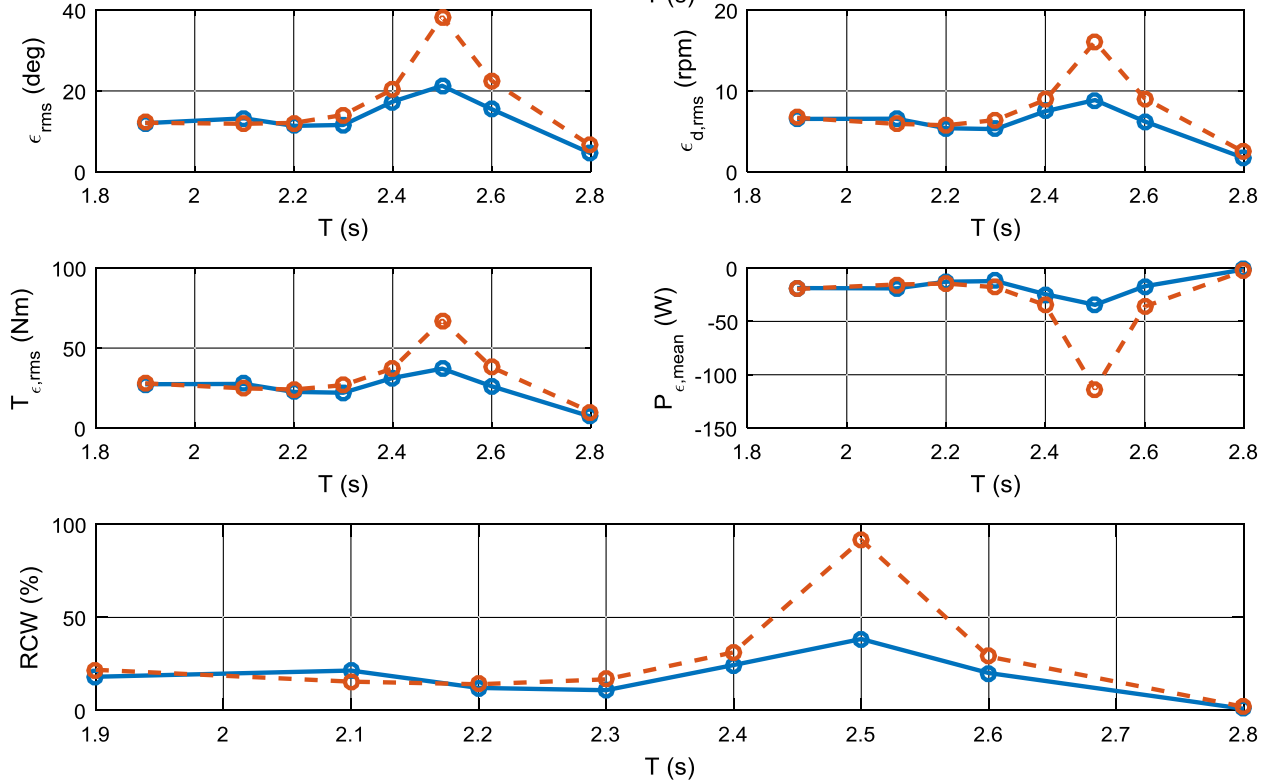

Fig. 11. Frequency sweep with constant PTO damping coefficient $(40 \mathrm{Nms} / \mathrm{rad})$.

varied between $1.9 \mathrm{~s}$ and $2.8 \mathrm{~s}$. The regular wave height was maintained constant at $0.15 \mathrm{~m}$. Figure 11 shows the comparison between the experimental and numerical root-mean-square values of the main physical quantities. In the case of the extracted power, the mean value is taken into account.

Considering the pitch motion, a good degree of accuracy is reached for all periods considered, despite an overestimation can be observed in correspondence of the resonance at $2.5 \mathrm{~s}$. This difference can be justified taking into account the linear nature of the hydrodynamic model, which does not contemplate the viscous effects.

Since the hull motions influence the motion of the inner pendulum, some discrepancies around the $2.5 \mathrm{~s}$ wave period can be observed also for the pendulum angular displacement and velocity, the torque at the PTO axis and the extracted power. In particular, in the case of the extracted power the deviation of the numerical result from the experimental one depends on the error of the torque and of the pendulum angular velocity. This is due to the fact that the power is calculated as the product between the torque and the angular velocity. 
Table 3. Mean relative error of the frequency sweep comparison.

\begin{tabular}{lcc}
\hline Physical quantity & Relative error (\%) & C.O.V. \\
\hline$\delta_{\text {rms }}$ & 10.45 & $3.31 \% / \mathrm{deg}$ \\
$\varepsilon_{\text {rms }}$ & 25.41 & $1.15 \% / \mathrm{deg}$ \\
$\dot{\varepsilon}_{\text {rms }}$ & 26.12 & $1.12 \% / \mathrm{rpm}$ \\
$T_{\varepsilon, \mathrm{rms}}$ & 25.93 & $1.13 \% / \mathrm{Nm}$ \\
$P_{\varepsilon, \mathrm{rms}}$ & 66.08 & $1.21 \% / \mathrm{W}$ \\
RCW & 48.85 & $1.12 \% / \%$ \\
\hline
\end{tabular}

In addition, the Relative Capture Width (RCW) (defined as the ratio between the extracted power and the wave power intercepted by the hull) computed with the numerical model do not give a good representation of the experimental data.

Table 3 reports the average value of the percentage relative error and the coefficient of variation (C.O.V.) evaluated for each physical quantity.

As stated before, the hydrodynamic model do not take into account the viscous effects, which can be relevant when motion with large velocity and amplitude occurs.

Such effects can be included in the numerical model in different ways, for example by using a calibration process based on experimental data or by using fully viscous simulations [Penalba et al., 2015]. In the case of this work, the first approach was adopted: the nonlinear effect proportional to the square of the velocity was introduced on the equation of the pitch motion and the viscous coefficient was identified thanks to a minimization procedure of the error between numerical and experimental data. The analytical expression of the nonlinear viscous effect on the pitch degree of freedom is given by Eq. (10.1), where $\beta$ is the viscous coefficient estimated through the identification procedure.

$$
F_{\text {vis }}=-\beta \dot{\delta}|\dot{\delta}|
$$

In Fig. 12 and in Table 4, the results obtained are summarized, showing a significant improvement of the numerical results quality.

\subsection{PTO damping coefficient}

The second result shown in this section is the comparison of the main physical quantities versus the PTO damping coefficient, considering constant wave period.

Figure 13 shows the results obtained with a wave period equal to $2.2 \mathrm{~s}$. In this condition, the root mean square values of the numerical results fit adequately the experimental data.

Furthermore, in correspondence of this wave period, increasing of the PTO damping coefficient it is possible to increase the performances of the WEC, obtaining a maximization of the mean power when this coefficient is equal to $600 \mathrm{Nms} / \mathrm{rad}$, both for the model and experiments. This value is the maximum of the evaluated range. 

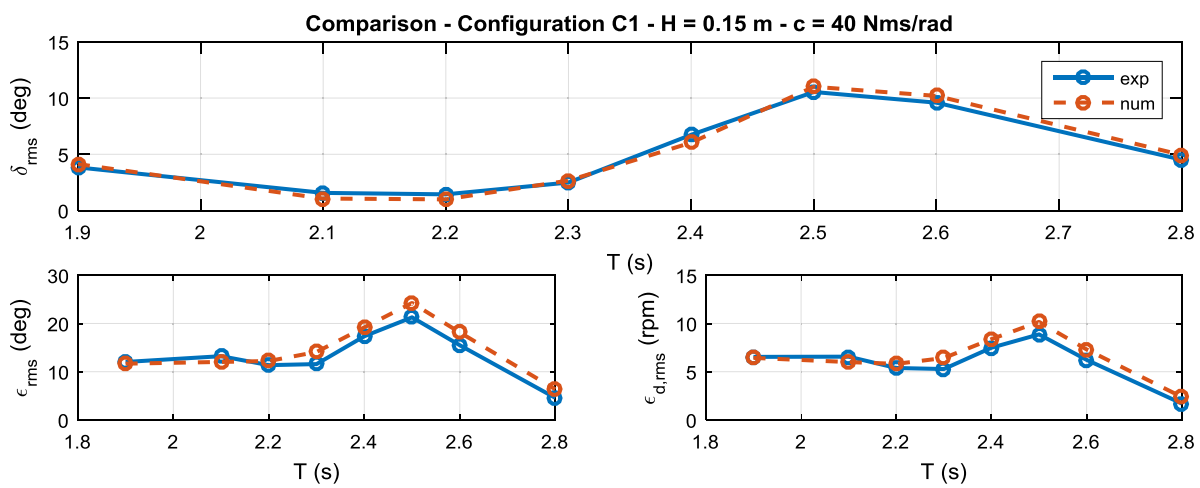

$\mathrm{T}(\mathrm{s})$
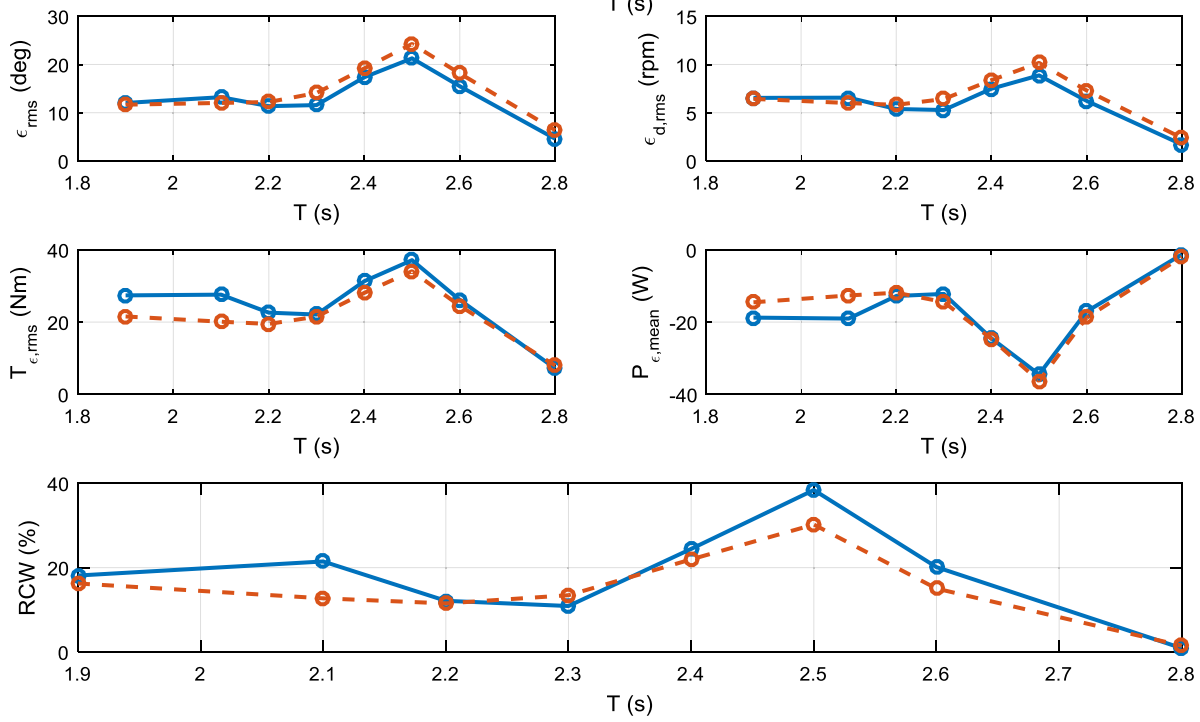

Fig. 12. Frequency sweep with constant PTO damping coefficient (40 Nms/rad) and viscous effect on pitch DOF.

Table 4. Mean relative error of the frequency sweep comparison.

\begin{tabular}{lcc}
\hline Physical quantity & Relative error (\%) & C.O.V. \\
\hline$\delta_{\mathrm{rms}}$ & -4.84 & $-2.06 \% / \mathrm{deg}$ \\
$\varepsilon_{\mathrm{rms}}$ & 5.47 & $3.67 \% / \mathrm{deg}$ \\
$\dot{\varepsilon}_{\mathrm{rms}}$ & 6.07 & $3.30 \% / \mathrm{rpm}$ \\
$T_{\varepsilon, \mathrm{rms}}$ & 5.89 & $3.37 \% / \mathrm{Nm}$ \\
$P_{\varepsilon, \mathrm{rms}}$ & 3.08 & $2.61 \% / \mathrm{W}$ \\
RCW & -2.74 & $-8.33 \% / \%$ \\
\hline
\end{tabular}

Table 5 describes the average value of the percentage relative error evaluated for each physical quantity.

The hydrodynamic model agreement on the pitch motion estimated during the wave tests at $2.5 \mathrm{~s}$ wave period is fulfilled, as shown in Fig. 14 and proved by the relative error between experimental and numerical data given in Table 6. However, an overestimation of the extracted power can be found: such discrepancy is induced by a not proper prediction of the pendulum angular velocity and of the torque at the PTO shaft. Here, the PTO bearing friction was not taken into account and this might be the cause of the overestimation pointed out above. 

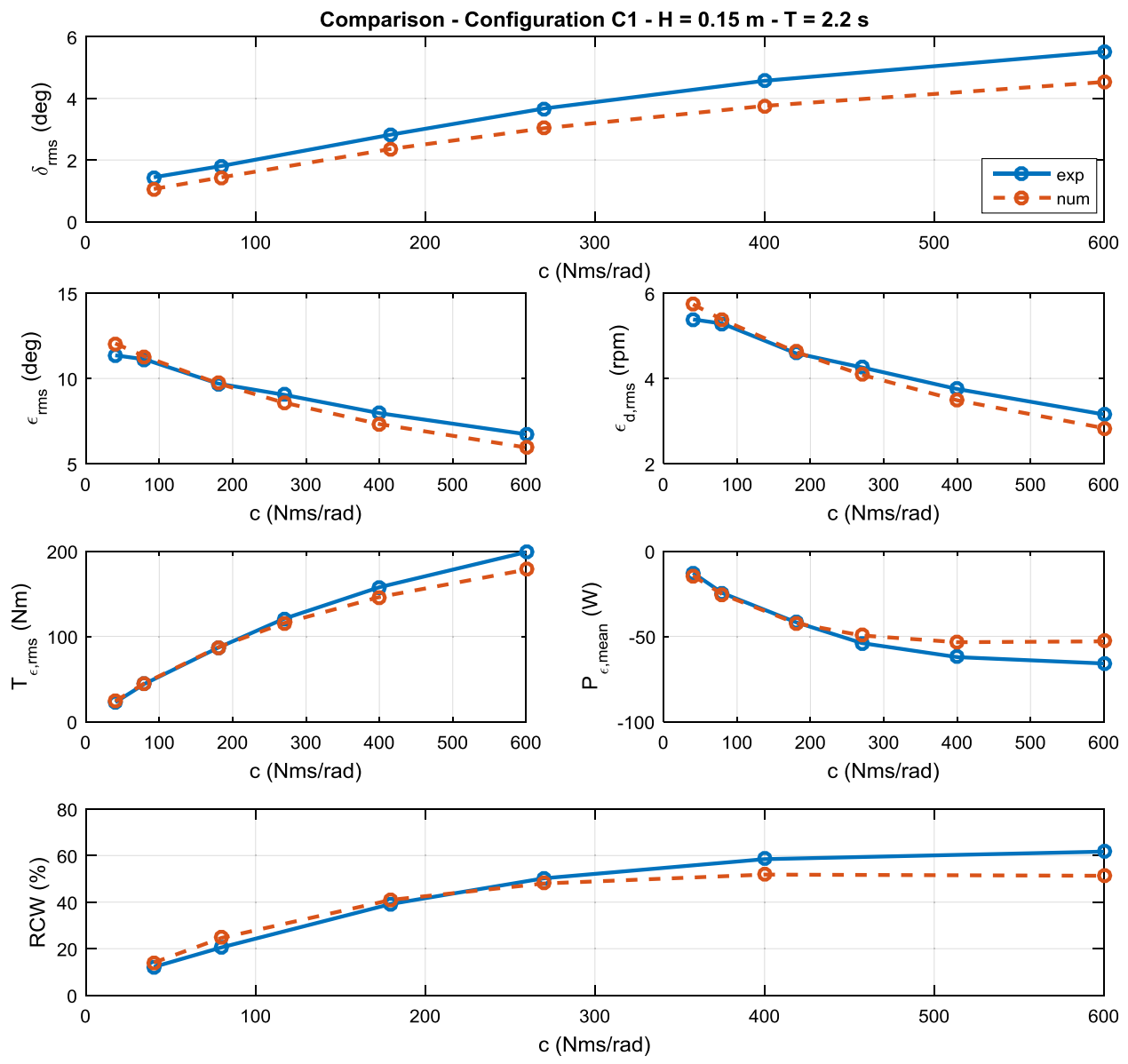

Fig. 13. Effect of damping coefficient on the PeWEC performances at the nominal wave period $2.2 \mathrm{~s}$.

Table 5. Mean relative error of the comparison varying the damping coefficient at the nominal wave period $2.2 \mathrm{~s}$.

\begin{tabular}{lcc}
\hline Physical quantity & Relative error $(\%)$ & C.O.V. \\
\hline$\delta_{\mathrm{rms}}$ & -10.25 & $-0.18 \% / \mathrm{deg}$ \\
$\varepsilon_{\mathrm{rms}}$ & -2.82 & $-2.34 \% / \mathrm{deg}$ \\
$\dot{\varepsilon}_{\mathrm{rms}}$ & -2.04 & $-3.02 \% / \mathrm{rpm}$ \\
$T_{\varepsilon, \mathrm{rms}}$ & -2.21 & $-2.08 \% / \mathrm{Nm}$ \\
$P_{\varepsilon, \mathrm{rms}}$ & 1.02 & $-2.99 \% / \mathrm{W}$ \\
$\mathrm{RCW}$ & 3.85 & $4.25 \% / \%$ \\
\hline
\end{tabular}

On the other hand, despite some differences between the numerical results and the experimental data, the trends predicted by the numerical model are in agreement with the real behavior of the system. 

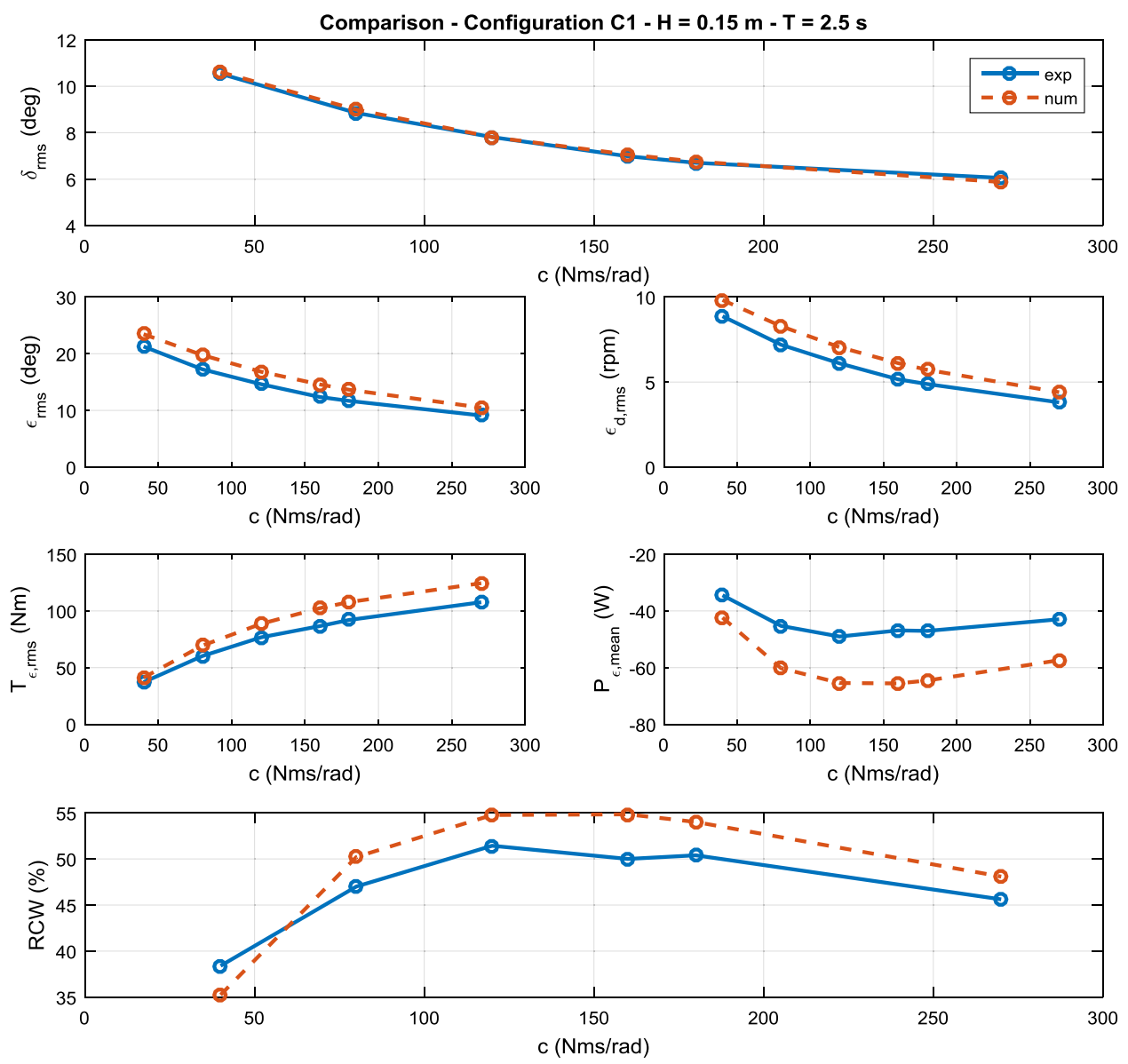

Fig. 14. Effect of damping coefficient on the PeWEC performances at the wave period $2.5 \mathrm{~s}$.

Table 6. Mean relative error of the comparison varying the damping coefficient at the wave period $2.5 \mathrm{~s}$.

\begin{tabular}{lcc}
\hline Physical quantity & Relative error (\%) & C.O.V \\
\hline$\delta_{\mathrm{rms}}$ & 0.205 & $7.94 \% / \mathrm{deg}$ \\
$\varepsilon_{\mathrm{rms}}$ & 14.98 & $0.18 \% / \mathrm{deg}$ \\
$\dot{\varepsilon}_{\mathrm{rms}}$ & 15.47 & $0.17 \% / \mathrm{rpm}$ \\
$T_{\varepsilon, \mathrm{rms}}$ & 15.45 & $0.16 \% / \mathrm{Nm}$ \\
$P_{\varepsilon, \mathrm{rms}}$ & 33.33 & $0.17 \% / \mathrm{W}$ \\
$\mathrm{RCW}$ & 4.56 & $1.41 \% / \%$ \\
\hline
\end{tabular}

\section{Conclusions}

The paper shows the development of the mathematical model of the PeWEC system.

The 1:12 scaled prototype designed at Politecnico di Torino was tested at the INSEAN wave basin and the experimental results are used in order to validate the 
numerical model previously developed. This step is performed in order to reliably design the full-scale device.

The numerical model was initially developed taking into account the linear Cummins' equation and a great overestimation of the pitch motion amplitude was pointed out. Then the hydrodynamic model was improved introducing the nonlinear viscous effect, which was identified on the base of the experimental data.

The agreement between numerical results and experimental data obtained after the numerical model tuning can be considered acceptable. Furthermore, it is important to highlight that the numerical trends are in accord with the experimental data, also in the case of wave frequencies closed to the natural frequency of the system.

In the end, it is possible to state that the numerical model can be used for the design of the full-scale device and for a deep parametrical analysis of the system.

Future work is oriented to the identification of the friction of the PTO bearings and the extension of the parametric study of the system. The aim of this study is to understand the role of the physical parameters of the device, such as the pendulum mass, length and inertia, the distance between the pendulum hinge and the COG of the structure, etc., on the performances of the device and relate them with the technical-economic feasibility.

\section{Acknowledgments}

This work was funded by the Italian Government through "Accordo di Programma ENEA-MiSE 2015".

\section{References}

Ansys [2013] "AQWA Theory Manual, Release 15.0," USA.

Airy, G. B. [1841] "Tides and Waves," pp. 396.

Baghfalaki, M., Das, S. K. and Das, S. N. [2012] "Analytical model to determine response amplitude operator of a floating body for coupled roll and yaw motions and frequency-based analysis," International Journal of Applied Mechanics 4(4), $1-20$.

Bracco, G., Giorcelli, E. and Mattiazzo, G. [2008] "One degree of freedom gyroscopic mechanism for wave energy converters," IDETC/CIE 2008, Brooklyn, New York, USA, August 3-6, 2008.

Bracco, G., Giorcelli, E. and Mattiazzo, G. [2010] "ISWEC design of a prototype model for wave tank test," ASME 2010 10th Biennal Conference on Engineering System Design and Analysis (ESDA 2010), Istanbul, Turkey, July 12-14, 2010.

Bracco, G., Giorcelli, E., Mattiazzo, G., Marignetti, F., Carbone, S. and Attaianese, C. [2011] "Design and experiments of linear tubular generators for the inertial sea wave energy converter, 2011 IEEE energy conversion congress and exposition," ECCE 2011, pp. 3864-3871.

Bracco, G., Giorcelli, E., Mattiazzo, G., Orlando, V. and Raffero, M. [2015a] "HardwareIn-the-Loop test rig for the ISWEC wave energy system," Mechatronics 25, $11-17$. 
Bracco, G., Giorcelli, E., Giorgi, G., Mattiazzo, G., Passione, B., Raffero, M. and Vissio, G. [2015b] "Performance assessment of the full scale Iswec system," ICIT 2015, Sevilla, 17-19 March 2015, pp. 2499-2505.

Bracco, G., Giorcelli, E., Mattiazzo, G., Passione, B., Prudentino, E., Raffero, M. and Vissio, G. [2015c] "Application of linear model predictive control to the ISWEC," Renewable Energies Offshore - $1^{\text {st }}$ International Conference on Renewable Energies Offshore, RENEW 2014, pp. 257.

Cagninei, A., Raffero, M., Bracco, G., Giorcelli, E., Mattiazzo, G. and Poggi, D. [2015] "Productivity analysis of the full scale inertial sea wave energy converter prototype: A test case in Pantelleria Island," Journal of Renewable Sustainable Energy 7, 061703.

Cordonnier, J., Gorintin, F., De Cagny, A., Clément, A. H. and Babarit, A. [2015] "SEAREV: Case study of the development of a wave energy converter," Renewable Energy 80, 40-52.

Cummins, W. E. [1962] "The impulse response function and ship motions," David Taylor model basin, Report 1661, Department of the Navy, Washington DC.

Engström, J., Eriksson, M., Isberg, J. and Leijon, M. [2009] "Wave energy converter with enhanced amplitude response at frequencies coinciding with Swedish west coast sea states by use of a supplementary submerged body," Journal of Applied Physics 106, 064512.

European Union Horizon 2020 "Secure, clean and efficient energy," https://ec.europa.eu/ programmes/horizon2020/en/h2020-section/secure-clean-and-efficient-energy, accessed May 2016.

Falcão, A. F. de O. [2010] "Wave energy utilization: A review of the technologies," Renewable and Sustainable Energy Reviews 14(3), 899-918.

Falcão, A. F. de O. [2013] "Modeling of wave energy conversion," Instituto Superior Técnico, Universidade Técnica de Lisboa.

Faltinsen, O. M. [1990] "Sea Loads on Ships and Offshore structures," Cambridge University Press, 1990.

French, M. J. [2006] "On the difficulty of inventing an economical sea wave energy converter: A personal view," 4 Haverbreaks Place, Lancaster LA1 5BH, UK.

Güney, M. S. [2015] "Wave energy systems," Journal of Naval Science and Engineering $\mathbf{1 1}(2), 25-51$.

Huijsmans, R. H. M. [1996] "Mathematical modeling of the mean wave drift force in current: A numerical and experimental study," MARIN.

INSEAN wave basin characteristics [2011] "International towing tank conference catalogue of facilities towing tanks, seakeeping and maneuvering basins," 23rd ITTC (annex to ITTC - news no. 44, June 2001).

Journée, J. M. J. and Massie, W. W. [2001] Offshore Hydromechanics, 1st edn. (Delft University of Technology).

Liberti, L., Carillo, A. and Sannino, G. [2013] "Wave energy resource assessment in the Mediterranean, the Italian perspective," Renewable Energy 50, 938-949.

López, I., Andreu, J., Ceballos, S., Martínez de Alegría, I. and Kortabarria, I. [2013] "Review of wave energy technologies and the necessary power-equipment," Renewable and Sustainable Energy Reviews 27, 413-434.

Ogilvie, T. F. [1964] "Recent progress towards the understanding and prediction of ship motions," Proceedings of the Fifth Symposium of Naval Hydrodynamics, Bergen, Norway, 1964, ACR-112, pp. 3-79, ONR.

Penalba, R. M., Giorgi, G. and Ringwood, J. [2015] "A review of nonlinear approaches for wave energy modeling," Proceedings of the 11th European Wave and Tidal Energy Conference, ISSN 2309-1983. 
Pinkster, J. A. [1980] "Low frequency second order wave exciting forces on floating structures," PhD Thesis, Department of Mechanical, Maritime and Materials Engineering, Delft University of Technology, Delft, Holland.

Pozzi, N. [2014] "Modeling and testing of a wave energy converter," Master Degree Thesis, Department of Mechanical and Aerospace Engineering, Politecnico di Torino, Torino, Italy.

Prins, H. [1995] "Time-Domain Calculations of Drift Forces and Moments," Thesis, Technische Univesiteit Delft.

Rinaldi, G., Fontanella, A., Sannino, G., Bracco, G., Giorcelli, E., Mattiazzo, G. and Bludszuweit, H. [2015] "Development of a simplified analytical model for a passive inertial system solicited by wave motion," International Journal of Marine Energy 13, $45-61$.

Sirigu, S. A., Vissio, G., Bracco, G., Giorcelli, E., Passione, B., Raffero, M. and Mattiazzo, G. [2016] "ISWEC design tool," International Journal of Marine Energy.

Solovyev, A. N. and Duong, L. V. [2016], "Optimization for the harvesting structure of the piezoelectric bimorph energy harvesters circular plate by reduced order finite element analysis," International Journal of Applied Mechanics 8(3), 1650029.

Trimble, A., Lang, J. H., Pabon, J. and Slocum, A. [2010] "A device for harvesting energy from rotational vibrations," ASME Journal of Mechanical Design 132(9), 091001.

US Department of Energy "Marine and hydrokinetic market acceleration and deployment," http://energy.gov/eere/water/marine-and-hydrokinetic-market-accelerationand-deployment\#ad, accessed May 2016.

WAMIT [2015] "WAMIT User Manual," USA.

Xie, L. and Cai, M. [2015] "Development of a suspended backpack for harvesting biomechanical energy," ASME Journal of Mechanical Design 137(5), 054503. 